\title{
Supplementary Material: Effects of vernal equinox solar eclipse on temperatures and wind directions in Switzerland
}

\author{
Werner Eugster ${ }^{1}$, Carmen Emmel ${ }^{1}$, Sebastian Wolf ${ }^{2}$, Nina Buchmann ${ }^{1}$, Joseph P. McFadden ${ }^{3}$, and \\ C. David Whiteman ${ }^{4}$ \\ ${ }^{1}$ ETH Zurich, Department of Environmental Systems Science, Institute of Agricultural Sciences, 8092 Zurich, Switzerland \\ ${ }^{2}$ ETH Zurich, Department of Environmental Systems Science, Institute of Terrestrial Ecosystems, 8092 Zurich, Switzerland \\ ${ }^{3}$ University of California, Santa Barbara, Department of Geography, Earth Research Institute, Santa Barbara, California, \\ 93106-4060, USA \\ ${ }^{4}$ University of Utah, Atmospheric Sciences Department, Salt Lake City, Utah, USA
}

Correspondence to: W. Eugster (eugsterw @ethz.ch)

\section{List of Tables}

S1 Swiss FluxNet sites with high-resolution $(20 \mathrm{~Hz})$ turbulent flux measurements and meteorological measurements at the temporal resolution specified in the last column. . . . . . . . . . . . . . . . . . . 2

S2 MeteoSwiss standard weather stations used in this study with temperature drop $\Delta T$ and timing $t(\Delta T)$ (hour:minute CET). Measurement interval was 10 minutes. . . . . . . . . . . . . . . . . . . . . . . . . . 2 15

S3 MeteoSwiss standard weather stations measured wind speed and wind direction and their classification according to topography. Where possible, the orientation of the valley and the slope angle are given. . . . . . . . . 6

\section{List of Figures}

S1 Wind direction evolution at MeteoSwiss sites classified as 'flat'. Black line and gray symbols: day of eclipse (20 March 2015). Light blue line and symbols (19 March 2015) and dark blue line and symbols (18 March 2015): conditions on the days before for comparison. The timing of the first (1) and last (4) contact and the eclipse maximum (M) are shown with vertical lines. Site names and codes correspond to those given in Table S3. The bold lines are local polynomial regression (loess) fits. Note that the y-axis was shifted by $0,90,180$ or

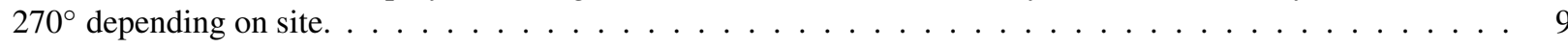

S2 As Figure S1, but for sites classified as 'valley' sites in rather small valleys (otherwise a site was classified as 'flat'). . . . . . . . . . . . . . . . . . . . . . . . . . 12

S3 As Figure S1, but for sites classified as 'mountain or hilltop' sites. . . . . . . . . . . . . . . . . . . 14

S4 As Figure S1, but for sites classified as 'slope' sites. . . . . . . . . . . . . . . . . . . . . . . . 17 
Table S1. Swiss FluxNet sites with high-resolution $(20 \mathrm{~Hz})$ turbulent flux measurements and meteorological measurements at the temporal resolution specified in the last column.

\begin{tabular}{llccccc}
\hline CODE & Site Name & Ecosystem Type & Latitude & Longitude & Elevation & Met Interval $^{a}$ \\
\hline CH-AWS & Alp Weissenstein & Alpine Pasture & $46^{\circ} 34^{\prime} 59.5^{\prime \prime} \mathrm{N}$ & $9^{\circ} 47^{\prime} 25.5^{\prime \prime} \mathrm{E}$ & $1978 \mathrm{~m}$ & $10 \mathrm{~min}$ \\
CH-CHA & Chamau & Valley Grassland & $47^{\circ} 12^{\prime} 36.8^{\prime \prime ~ N}$ & $8^{\circ} 24^{\prime} 37.6^{\prime \prime} \mathrm{E}$ & $393 \mathrm{~m}$ & $30 \mathrm{~min}$ \\
CH-DAV & Davos & Deciduous Forest & $46^{\circ} 48^{\prime} 55.2^{\prime \prime} \mathrm{N}$ & $9^{\circ} 51^{\prime} 21.3^{\prime \prime} \mathrm{E}$ & $1639 \mathrm{~m}$ & $1 \mathrm{~min} / 10 \mathrm{~min}$ \\
CH-FRU & Früebüel & Montane Grassland & $47^{\circ} 06^{\prime} 57.0^{\prime \prime} \mathrm{N}$ & $8^{\circ} 32^{\prime} 16.0^{\prime \prime} \mathrm{E}$ & $982 \mathrm{~m}$ & $30 \mathrm{~min}$ \\
CH-LAE & Lägeren & Mixed Forest & $47^{\circ} 28^{\prime} 42.0^{\prime \prime} \mathrm{N}$ & $8^{\circ} 21^{\prime} 51.8^{\prime \prime} \mathrm{E}$ & $682 \mathrm{~m}$ & $30 \mathrm{~min} / 10 \mathrm{~min}$ \\
$\mathrm{CH}-\mathrm{OE} 2$ & Oensingen & Cropland & $47^{\circ} 17^{\prime} 11.1^{\prime \prime} \mathrm{N}$ & $7^{\circ} 44^{\prime} 01.5^{\prime \prime} \mathrm{E}$ & $452 \mathrm{~m}$ & $1 \mathrm{~min}$ \\
\hline
\end{tabular}

$a$ if two averaging intervals are given, then the first relates to all-wave radiation measurements and the second to temperature and other standard meteorological measurements during the 2015 eclipse

Table S2. MeteoSwiss standard weather stations used in this study with temperature drop $\Delta T$ and timing $t(\Delta T)$ (hour:minute CET). Measurement interval was 10 minutes.

\begin{tabular}{|c|c|c|c|c|c|c|}
\hline CODE & Station Name & Elevation (m) & Longitude (E) & Latitude $(\mathrm{N})$ & $\Delta T(\mathrm{~K})$ & $t(\Delta T)$ \\
\hline $\mathrm{ABO}$ & Adelboden & 1326 & $7^{\circ} 33^{\prime} 37.5^{\prime \prime}$ & $46^{\circ} 29^{\prime} 32.1^{\prime \prime}$ & -1.56 & $09: 50$ \\
\hline AIG & Aigle & 381 & $6^{\circ} 55^{\prime} 27.9^{\prime \prime}$ & $46^{\circ} 19^{\prime} 35.9^{\prime \prime}$ & -1.05 & 10:00 \\
\hline ALT & Altdorf & 438 & $8^{\circ} 37^{\prime} 18.5^{\prime \prime}$ & $46^{\circ} 53^{\prime} 13.3^{\prime \prime}$ & -2.89 & 09:40 \\
\hline AND & Andeer & 987 & $9^{\circ} 25^{\prime} 54.9^{\prime \prime}$ & $46^{\circ} 36^{\prime} 36.4^{\prime \prime}$ & -0.49 & $10: 20$ \\
\hline ANT & Andermatt & 1438 & $8^{\circ} 34^{\prime} 50.0^{\prime \prime}$ & $46^{\circ} 37^{\prime} 51.3^{\prime \prime}$ & -1.32 & $09: 30$ \\
\hline ARD & Arosa & 1840 & $9^{\circ} 40^{\prime} 28.3^{\prime \prime}$ & $46^{\circ} 46^{\prime} 45.0^{\prime \prime}$ & -3.82 & $09: 40$ \\
\hline ARH & Altenrhein & 398 & $9^{\circ} 34^{\prime} 00.5^{\prime \prime}$ & $47^{\circ} 29^{\prime} 01.2^{\prime \prime}$ & -1.35 & $10: 20$ \\
\hline ATT & Les Attelas & 2730 & $7^{\circ} 16^{\prime} 07.5^{\prime \prime}$ & $46^{\circ} 05^{\prime} 56.8^{\prime \prime}$ & -0.46 & $10: 30$ \\
\hline BAS & Basel / Binningen & 316 & $7^{\circ} 35^{\prime} 00.8^{\prime \prime}$ & $47^{\circ} 32^{\prime} 27.7^{\prime \prime}$ & -0.32 & $09: 30$ \\
\hline BEH & Passo del Bernina & 2260 & $10^{\circ} 01^{\prime} 10.5^{\prime \prime}$ & $46^{\circ} 24^{\prime} 33.0^{\prime \prime}$ & -0.98 & $10: 20$ \\
\hline BER & Bern / Zollikofen & 552 & $7^{\circ} 27^{\prime} 50.4^{\prime \prime}$ & $46^{\circ} 59^{\prime} 26.7^{\prime \prime}$ & -1.46 & $09: 50$ \\
\hline BEZ & Beznau & 325 & $8^{\circ} 13^{\prime} 59.8^{\prime \prime}$ & $47^{\circ} 33^{\prime} 26.0^{\prime \prime}$ & -0.77 & 10:00 \\
\hline BIE & Bière & 683 & $6^{\circ} 20^{\prime} 32.6^{\prime \prime}$ & $46^{\circ} 31^{\prime} 29.5^{\prime \prime}$ & -1.90 & 09:40 \\
\hline BIN & Binn & 1448 & $8^{\circ} 11^{\prime} 27.3^{\prime \prime}$ & $46^{\circ} 21^{\prime} 55.7^{\prime \prime}$ & -2.57 & 09:50 \\
\hline BIZ & Bischofszell & 470 & $9^{\circ} 14^{\prime} 05.0^{\prime \prime}$ & $47^{\circ} 29^{\prime} 50.6^{\prime \prime}$ & -1.16 & 10:00 \\
\hline BLA & Blatten, Lötschental & 1538 & $7^{\circ} 49^{\prime} 23.5^{\prime \prime}$ & $46^{\circ} 25^{\prime} 13.6^{\prime \prime}$ & -0.87 & 10:10 \\
\hline BOL & Boltigen & 820 & $7^{\circ} 23^{\prime} 03.1^{\prime \prime}$ & $46^{\circ} 37^{\prime} 24.7^{\prime \prime}$ & -1.11 & 09:50 \\
\hline BOU & Bouveret & 374 & $6^{\circ} 51^{\prime} 25.2^{\prime \prime}$ & $46^{\circ} 23^{\prime} 36.4^{\prime \prime}$ & -1.63 & $09: 10$ \\
\hline BRL & La Brévine & 1050 & $6^{\circ} 36^{\prime} 37.0^{\prime \prime}$ & $46^{\circ} 59^{\prime} 01.8^{\prime \prime}$ & -2.71 & $09: 20$ \\
\hline BUF & Buffalora & 1968 & $10^{\circ} 16^{\prime} 01.9^{\prime \prime}$ & $46^{\circ} 38^{\prime} 53.3^{\prime \prime}$ & -1.85 & $09: 40$ \\
\hline BUS & Buchs / Aarau & 386 & $8^{\circ} 04^{\prime} 46.0^{\prime \prime}$ & $47^{\circ} 23^{\prime} 03.6^{\prime \prime}$ & -1.47 & 09:40 \\
\hline $\mathrm{CDF}$ & La Chaux-de-Fonds & 1018 & $6^{\circ} 47^{\prime} 32.3^{\prime \prime}$ & $47^{\circ} 04^{\prime} 58.6^{\prime \prime}$ & -2.71 & $09: 40$ \\
\hline CEV & Cevio & 417 & $8^{\circ} 36^{\prime} 11.4^{\prime \prime}$ & $46^{\circ} 19^{\prime} 13.8^{\prime \prime}$ & -0.20 & $10: 20$ \\
\hline CGI & Nyon / Changins & 455 & $6^{\circ} 13^{\prime} 39.9^{\prime \prime}$ & $46^{\circ} 24^{\prime} 03.7^{\prime \prime}$ & -1.41 & $09: 20$ \\
\hline $\mathrm{CHA}$ & Chasseral & 1599 & $7^{\circ} 03^{\prime} 15.8^{\prime \prime}$ & $47^{\circ} 07^{\prime} 54.4^{\prime \prime}$ & -0.92 & $09: 40$ \\
\hline CHB & Les Charbonnières & 1045 & $6^{\circ} 18^{\prime} 44.7^{\prime \prime}$ & $46^{\circ} 40^{\prime} 12.5^{\prime \prime}$ & -2.75 & $09: 40$ \\
\hline $\mathrm{CHD}$ & Château-d'Oex & 1029 & $7^{\circ} 08^{\prime} 22.7^{\prime \prime}$ & $46^{\circ} 28^{\prime} 47.3^{\prime \prime}$ & -1.25 & 10:00 \\
\hline CHM & Chaumont & 1136 & $6^{\circ} 58^{\prime} 43.7^{\prime \prime}$ & $47^{\circ} 02^{\prime} 57.0^{\prime \prime}$ & -1.71 & $09: 40$ \\
\hline $\mathrm{CHU}$ & Chur & 556 & $9^{\circ} 31^{\prime} 50.1^{\prime \prime}$ & $46^{\circ} 52^{\prime} 13.5^{\prime \prime}$ & -1.40 & 10:00 \\
\hline $\mathrm{CHZ}$ & Cham & 442 & $8^{\circ} 27^{\prime} 52.7^{\prime \prime}$ & $47^{\circ} 11^{\prime} 17.8^{\prime \prime}$ & -0.59 & 10:00 \\
\hline CIM & Cimetta & 1661 & $8^{\circ} 47^{\prime} 29.9^{\prime \prime}$ & $46^{\circ} 12^{\prime} 01.5^{\prime \prime}$ & -1.09 & $09: 30$ \\
\hline CMA & Crap Masegn & 2480 & $9^{\circ} 10^{\prime} 48.0^{\prime \prime}$ & $46^{\circ} 50^{\prime} 32.3^{\prime \prime}$ & -0.01 & $10: 20$ \\
\hline $\mathrm{COM}$ & Acquarossa / Comprovasco & 575 & $8^{\circ} 56^{\prime} 08.4^{\prime \prime}$ & $46^{\circ} 27^{\prime} 33.9^{\prime \prime}$ & -1.18 & 10:00 \\
\hline $\mathrm{COV}$ & Piz Corvatsch & 3302 & $9^{\circ} 49^{\prime} 16.5^{\prime \prime}$ & $46^{\circ} 25^{\prime} 04.8^{\prime \prime}$ & -0.85 & $09: 50$ \\
\hline $\mathrm{COY}$ & Courtelary & 695 & $7^{\circ} 05^{\prime} 26.3^{\prime \prime}$ & $47^{\circ} 10^{\prime} 50.9^{\prime \prime}$ & -1.72 & 10:00 \\
\hline CRM & Cressier & 431 & $7^{\circ} 03^{\prime} 32.8^{\prime \prime}$ & $47^{\circ} 02^{\prime} 51.4^{\prime \prime}$ & -0.54 & $09: 40$ \\
\hline DAV & Davos & 1594 & $9^{\circ} 50^{\prime} 36.6^{\prime \prime}$ & $46^{\circ} 48^{\prime} 46.6^{\prime \prime}$ & -2.30 & $09: 40$ \\
\hline DEM & Delémont & 439 & $7^{\circ} 20^{\prime} 58.4^{\prime \prime}$ & $47^{\circ} 21^{\prime} 06.1^{\prime \prime}$ & -1.48 & $10: 40$ \\
\hline
\end{tabular}


Table S2. (continued)

\begin{tabular}{|c|c|c|c|c|c|c|}
\hline CODE & Station Name & Elevation (m) & Longitude (E) & Latitude (N) & $\Delta T(\mathrm{~K})$ & $t(\Delta T)$ \\
\hline DIA & Les Diablerets & 2964 & $7^{\circ} 12^{\prime} 13.6^{\prime \prime}$ & $46^{\circ} 19^{\prime} 36.3^{\prime \prime}$ & -0.95 & $09: 40$ \\
\hline DIS & Disentis / Sedrun & 1197 & $8^{\circ} 51^{\prime} 12.4^{\prime \prime}$ & $46^{\circ} 42^{\prime} 23.5^{\prime \prime}$ & -2.30 & $10: 00$ \\
\hline DOL & La Dôle & 1669 & $6^{\circ} 05^{\prime} 58.1^{\prime \prime}$ & $46^{\circ} 25^{\prime} 28.9^{\prime \prime}$ & -0.26 & $10: 30$ \\
\hline EBK & Ebnat-Kappel & 623 & $9^{\circ} 06^{\prime} 30.5^{\prime \prime}$ & $47^{\circ} 16^{\prime} 24.2^{\prime \prime}$ & -0.87 & $10: 10$ \\
\hline EGH & Eggishorn & 2893 & $8^{\circ} 05^{\prime} 33.8^{\prime \prime}$ & $46^{\circ} 25^{\prime} 35.5^{\prime \prime}$ & -2.19 & $09: 30$ \\
\hline EGO & Egolzwil & 521 & $8^{\circ} 00^{\prime} 17.1^{\prime \prime}$ & $47^{\circ} 10^{\prime} 45.9^{\prime \prime}$ & -1.27 & 09:50 \\
\hline EIN & Einsiedeln & 910 & $8^{\circ} 45^{\prime} 23.6^{\prime \prime}$ & $47^{\circ} 07^{\prime} 58.9^{\prime \prime}$ & -0.92 & $10: 20$ \\
\hline ELM & Elm & 958 & $9^{\circ} 10^{\prime} 31.3^{\prime \prime}$ & $46^{\circ} 55^{\prime} 25.5^{\prime \prime}$ & -1.84 & $10: 00$ \\
\hline ENG & Engelberg & 1035 & $8^{\circ} 24^{\prime} 37.6^{\prime \prime}$ & $46^{\circ} 49^{\prime} 18.8^{\prime \prime}$ & -3.08 & 09:50 \\
\hline EVI & Evionnaz & 482 & $7^{\circ} 01^{\prime} 36.3^{\prime \prime}$ & $46^{\circ} 10^{\prime} 58.6^{\prime \prime}$ & -0.10 & 09:50 \\
\hline EVO & Evolène / Villa & 1825 & $7^{\circ} 30^{\prime} 31.2^{\prime \prime}$ & $46^{\circ} 06^{\prime} 43.7^{\prime \prime}$ & -2.93 & $10: 00$ \\
\hline FAH & Fahy & 596 & $6^{\circ} 56^{\prime} 27.9^{\prime \prime}$ & $47^{\circ} 25^{\prime} 25.7^{\prime \prime}$ & -2.28 & $10: 00$ \\
\hline FLU & Flühli, LU & 939 & $8^{\circ} 01^{\prime} 13.2^{\prime \prime}$ & $46^{\circ} 53^{\prime} 22.0^{\prime \prime}$ & -1.24 & $10: 00$ \\
\hline FRE & Bullet / La Frétaz & 1205 & $6^{\circ} 34^{\prime} 34.7^{\prime \prime}$ & $46^{\circ} 50^{\prime} 26.2^{\prime \prime}$ & -1.82 & 09:40 \\
\hline GEN & Monte Generoso & 1600 & $9^{\circ} 01^{\prime} 04.3^{\prime \prime}$ & $45^{\circ} 55^{\prime} 39.3^{\prime \prime}$ & -0.85 & 09:40 \\
\hline GES & Gersau & 519 & $8^{\circ} 31^{\prime} 24.4^{\prime \prime}$ & $46^{\circ} 59^{\prime} 45.8^{\prime \prime}$ & -0.44 & 09:50 \\
\hline GIH & Giswil & 471 & $8^{\circ} 11^{\prime} 24.7^{\prime \prime}$ & $46^{\circ} 50^{\prime} 58.2^{\prime \prime}$ & -1.79 & 09:40 \\
\hline GLA & Glarus & 516 & $9^{\circ} 04^{\prime} 00.9^{\prime \prime}$ & $47^{\circ} 02^{\prime} 04.5^{\prime \prime}$ & -2.22 & 09:50 \\
\hline GOA & Göscheneralp & 1745 & $8^{\circ} 29^{\prime} 59.9^{\prime \prime}$ & $46^{\circ} 38^{\prime} 50.7^{\prime \prime}$ & -2.12 & 09:30 \\
\hline GOE & Gösgen & 380 & $7^{\circ} 58^{\prime} 25.2^{\prime \prime}$ & $47^{\circ} 21^{\prime} 46.9^{\prime \prime}$ & -0.94 & 09:40 \\
\hline GOR & Gornergrat & 3129 & $7^{\circ} 47^{\prime} 08.7^{\prime \prime}$ & $45^{\circ} 59^{\prime} 01.1^{\prime \prime}$ & -0.48 & 09:40 \\
\hline GRA & Fribourg / Posieux & 646 & $7^{\circ} 06^{\prime} 49.3^{\prime \prime}$ & $46^{\circ} 46^{\prime} 17.0^{\prime \prime}$ & -0.96 & 09:50 \\
\hline GRC & Grächen & 1605 & $7^{\circ} 50^{\prime} 12.5^{\prime \prime}$ & $46^{\circ} 11^{\prime} 43.1^{\prime \prime}$ & -0.48 & 09:50 \\
\hline GRE & Grenchen & 430 & $7^{\circ} 24^{\prime} 54.4^{\prime \prime}$ & $47^{\circ} 10^{\prime} 44.7^{\prime \prime}$ & -1.37 & 09:50 \\
\hline GRH & Grimsel Hospiz & 1980 & $8^{\circ} 19^{\prime} 59.7^{\prime \prime}$ & $46^{\circ} 34^{\prime} 18.1^{\prime \prime}$ & 0.03 & 09:50 \\
\hline GRO & Grono & 323 & $9^{\circ} 09^{\prime} 49.5^{\prime \prime}$ & $46^{\circ} 15^{\prime} 18.3^{\prime \prime}$ & -0.34 & $10: 00$ \\
\hline GSB & Col du Grand St-Bernard & 2472 & $7^{\circ} 10^{\prime} 14.9^{\prime \prime}$ & $45^{\circ} 52^{\prime} 07.6^{\prime \prime}$ & -1.67 & 09:50 \\
\hline GUE & Gütsch ob Andermatt & 2283 & $8^{\circ} 36^{\prime} 54.2^{\prime \prime}$ & $46^{\circ} 39^{\prime} 08.8^{\prime \prime}$ & -0.37 & $10: 00$ \\
\hline GUT & Güttingen & 440 & $9^{\circ} 16^{\prime} 45.8^{\prime \prime}$ & $47^{\circ} 36^{\prime} 06.1^{\prime \prime}$ & -1.25 & 09:50 \\
\hline GVE & Genève-Cointrin & 412 & $6^{\circ} 07^{\prime} 39.9^{\prime \prime}$ & $46^{\circ} 14^{\prime} 51.0^{\prime \prime}$ & -0.81 & 09:30 \\
\hline HAI & Salen-Reutenen & 718 & $9^{\circ} 01^{\prime} 26.1^{\prime \prime}$ & $47^{\circ} 39^{\prime} 04.4^{\prime \prime}$ & -2.32 & 09:50 \\
\hline HLL & Hallau & 419 & $8^{\circ} 28^{\prime} 13.7^{\prime \prime}$ & $47^{\circ} 41^{\prime} 50.2^{\prime \prime}$ & -1.08 & 09:50 \\
\hline HOE & Hörnli & 1144 & $8^{\circ} 56^{\prime} 29.9^{\prime \prime}$ & $47^{\circ} 22^{\prime} 15.1^{\prime \prime}$ & -2.40 & $10: 00$ \\
\hline ILZ & Ilanz & 698 & $9^{\circ} 12^{\prime} 55.2^{\prime \prime}$ & $46^{\circ} 46^{\prime} 30.1^{\prime \prime}$ & -0.77 & 09:50 \\
\hline INT & Interlaken & 577 & $7^{\circ} 52^{\prime} 12.5^{\prime \prime}$ & $46^{\circ} 40^{\prime} 20.1^{\prime \prime}$ & -2.29 & $09: 50$ \\
\hline JUN & Jungfraujoch & 3580 & $7^{\circ} 59^{\prime} 07.2^{\prime \prime}$ & $46^{\circ} 32^{\prime} 50.8^{\prime \prime}$ & -0.68 & $10: 00$ \\
\hline KLO & Zürich / Kloten & 426 & $8^{\circ} 32^{\prime} 09.9^{\prime \prime}$ & $47^{\circ} 28^{\prime} 46.6^{\prime \prime}$ & -1.49 & 09:50 \\
\hline KOP & Koppigen & 484 & $7^{\circ} 36^{\prime} 19.7^{\prime \prime}$ & $47^{\circ} 07^{\prime} 07.9^{\prime \prime}$ & -2.20 & $09: 40$ \\
\hline LAE & Lägern & 845 & $8^{\circ} 23^{\prime} 50.0^{\prime \prime}$ & $47^{\circ} 28^{\prime} 55.0^{\prime \prime}$ & -0.21 & $10: 10$ \\
\hline LAG & Langnau i.E. & 745 & $7^{\circ} 48^{\prime} 23.2^{\prime \prime}$ & $46^{\circ} 56^{\prime} 22.7^{\prime \prime}$ & -1.33 & 10:00 \\
\hline LEI & Leibstadt & 341 & $8^{\circ} 11^{\prime} 17.7^{\prime \prime}$ & $47^{\circ} 35^{\prime} 50.2^{\prime \prime}$ & -1.03 & 09:50 \\
\hline LUG & Lugano & 273 & $8^{\circ} 57^{\prime} 37.1^{\prime \prime}$ & $46^{\circ} 00^{\prime} 15.2^{\prime \prime}$ & -0.14 & 09:40 \\
\hline LUZ & Luzern & 454 & $8^{\circ} 18^{\prime} 03.5^{\prime \prime}$ & $47^{\circ} 02^{\prime} 11.2^{\prime \prime}$ & -0.34 & $10: 20$ \\
\hline MAG & Magadino / Cadenazzo & 203 & $8^{\circ} 56^{\prime} 01.2^{\prime \prime}$ & $46^{\circ} 09^{\prime} 36.1^{\prime \prime}$ & -0.48 & $10: 10$ \\
\hline MAH & Mathod & 437 & $6^{\circ} 34^{\prime} 04.7^{\prime \prime}$ & $46^{\circ} 44^{\prime} 13.1^{\prime \prime}$ & -2.30 & 09:50 \\
\hline MAS & Marsens & 714 & $7^{\circ} 04^{\prime} 10.8^{\prime \prime}$ & $46^{\circ} 39^{\prime} 23.3^{\prime \prime}$ & -1.48 & $09: 40$ \\
\hline MER & Meiringen & 588 & $8^{\circ} 10^{\prime} 09.3^{\prime \prime}$ & $46^{\circ} 43^{\prime} 56.0^{\prime \prime}$ & -1.08 & $09: 40$ \\
\hline MLS & Le Moléson & 1974 & $7^{\circ} 01^{\prime} 04.1^{\prime \prime}$ & $46^{\circ} 32^{\prime} 46.2^{\prime \prime}$ & -1.09 & 09:30 \\
\hline MOA & Mosen & 453 & $8^{\circ} 13^{\prime} 58.1^{\prime \prime}$ & $47^{\circ} 14^{\prime} 37.8^{\prime \prime}$ & -0.72 & 09:50 \\
\hline MOE & Möhlin & 344 & $7^{\circ} 52^{\prime} 40.5^{\prime \prime}$ & $47^{\circ} 34^{\prime} 19.9^{\prime \prime}$ & -1.23 & $10: 00$ \\
\hline MRP & Monte Rosa-Plattje & 2885 & $7^{\circ} 48^{\prime} 52.5^{\prime \prime}$ & $45^{\circ} 57^{\prime} 23.9^{\prime \prime}$ & -1.61 & $10: 10$ \\
\hline MTR & Matro & 2171 & $8^{\circ} 55^{\prime} 29.1^{\prime \prime}$ & $46^{\circ} 24^{\prime} 36.4^{\prime \prime}$ & -0.03 & 09:50 \\
\hline MUB & Mühleberg & 479 & $7^{\circ} 16^{\prime} 41.3^{\prime \prime}$ & $46^{\circ} 58^{\prime} 23.7^{\prime \prime}$ & -0.60 & 09:40 \\
\hline MVE & Montana & 1427 & $7^{\circ} 27^{\prime} 38.8^{\prime \prime}$ & $46^{\circ} 17^{\prime} 55.5^{\prime \prime}$ & -1.58 & $10: 00$ \\
\hline NAP & Napf & 1403 & $7^{\circ} 56^{\prime} 24.2^{\prime \prime}$ & $47^{\circ} 00^{\prime} 16.8^{\prime \prime}$ & -0.58 & $08: 50$ \\
\hline NAS & Naluns / Schlivera & 2400 & $10^{\circ} 15^{\prime} 41.0^{\prime \prime}$ & $46^{\circ} 49^{\prime} 01.8^{\prime \prime}$ & -0.09 & $08: 50$ \\
\hline NEU & Neuchâtel & 485 & $6^{\circ} 57^{\prime} 14.8^{\prime \prime}$ & $47^{\circ} 00^{\prime} 01.6^{\prime \prime}$ & -1.75 & $10: 20$ \\
\hline
\end{tabular}


Table S2. (continued)

\begin{tabular}{|c|c|c|c|c|c|c|}
\hline CODE & Station Name & Elevation (m) & Longitude (E) & Latitude (N) & $\Delta T(\mathrm{~K})$ & $t(\Delta T)$ \\
\hline ORO & Oron & 827 & $6^{\circ} 51^{\prime} 29.6^{\prime \prime}$ & $46^{\circ} 34^{\prime} 20.1^{\prime \prime}$ & -1.82 & $09: 50$ \\
\hline OTL & Locarno / Monti & 366 & $8^{\circ} 47^{\prime} 14.7^{\prime \prime}$ & $46^{\circ} 10^{\prime} 21.2^{\prime \prime}$ & -0.22 & 09:40 \\
\hline PAY & Payerne & 490 & $6^{\circ} 56^{\prime} 32.7^{\prime \prime}$ & $46^{\circ} 48^{\prime} 41.7^{\prime \prime}$ & -2.19 & $10: 10$ \\
\hline PIL & Pilatus & 2106 & $8^{\circ} 15^{\prime} 08.4^{\prime \prime}$ & $46^{\circ} 58^{\prime} 43.9^{\prime \prime}$ & -2.68 & 09:40 \\
\hline PIO & Piotta & 990 & $8^{\circ} 41^{\prime} 17.3^{\prime \prime}$ & $46^{\circ} 30^{\prime} 53.2^{\prime \prime}$ & -0.91 & $10: 30$ \\
\hline PLF & Plaffeien & 1042 & $7^{\circ} 15^{\prime} 57.6^{\prime \prime}$ & $46^{\circ} 44^{\prime} 51.6^{\prime \prime}$ & -2.21 & 09:50 \\
\hline PMA & Piz Martegnas & 2670 & $9^{\circ} 31^{\prime} 46.4^{\prime \prime}$ & $46^{\circ} 34^{\prime} 37.9^{\prime \prime}$ & -1.97 & 09:00 \\
\hline PSI & PSI Würenlingen & 334 & $8^{\circ} 13^{\prime} 45.9^{\prime \prime}$ & $47^{\circ} 32^{\prime} 18.4^{\prime \prime}$ & -1.87 & $10: 00$ \\
\hline PUY & Pully & 455 & $6^{\circ} 40^{\prime} 02.7^{\prime \prime}$ & $46^{\circ} 30^{\prime} 44.3^{\prime \prime}$ & -0.44 & 09:40 \\
\hline RAG & Bad Ragaz & 496 & $9^{\circ} 30^{\prime} 09.3^{\prime \prime}$ & $47^{\circ} 00^{\prime} 59.9^{\prime \prime}$ & -0.55 & 09:50 \\
\hline REH & Zürich / Affoltern & 443 & $8^{\circ} 31^{\prime} 04.4^{\prime \prime}$ & $47^{\circ} 25^{\prime} 39.6^{\prime \prime}$ & -0.08 & $10: 40$ \\
\hline ROB & Poschiavo / Robbia & 1078 & $10^{\circ} 3^{\prime} 40.2^{\prime \prime}$ & $46^{\circ} 20^{\prime} 47.9^{\prime \prime}$ & -0.70 & $10: 40$ \\
\hline ROE & Robièi & 1896 & $8^{\circ} 30^{\prime} 48.2^{\prime \prime}$ & $46^{\circ} 26^{\prime} 35.0^{\prime \prime}$ & -2.13 & 09:30 \\
\hline ROG & Rossberg & 1119 & $8^{\circ} 32^{\prime} 51.4^{\prime \prime}$ & $47^{\circ} 04^{\prime} 24.8^{\prime \prime}$ & 0.23 & $09: 50$ \\
\hline RUE & Rünenberg & 611 & $7^{\circ} 52^{\prime} 45.6^{\prime \prime}$ & $47^{\circ} 26^{\prime} 04.4^{\prime \prime}$ & -1.67 & 09:40 \\
\hline SAE & Säntis & 2502 & $9^{\circ} 20^{\prime} 37.1^{\prime \prime}$ & $47^{\circ} 14^{\prime} 58.0^{\prime \prime}$ & -0.94 & $09: 50$ \\
\hline SAG & Sattel-Aegeri & 790 & $8^{\circ} 38^{\prime} 13.1^{\prime \prime}$ & $47^{\circ} 04^{\prime} 51.8^{\prime \prime}$ & -2.34 & 09:50 \\
\hline SAM & Samedan & 1708 & $9^{\circ} 52^{\prime} 44.3^{\prime \prime}$ & $46^{\circ} 31^{\prime} 35.0^{\prime \prime}$ & -1.02 & 09:50 \\
\hline SBE & S. Bernardino & 1638 & $9^{\circ} 11^{\prime} 04.8^{\prime \prime}$ & $46^{\circ} 27^{\prime} 48.8^{\prime \prime}$ & -1.23 & 09:50 \\
\hline SBO & Stabio & 353 & $8^{\circ} 55^{\prime} 56.5^{\prime \prime}$ & $45^{\circ} 50^{\prime} 36.2^{\prime \prime}$ & -1.74 & 09:40 \\
\hline SCU & Scuol & 1303 & $10^{\circ} 16^{\prime} 59.6^{\prime \prime}$ & $46^{\circ} 47^{\prime} 35.8^{\prime \prime}$ & -2.18 & 09:40 \\
\hline SHA & Schaffhausen & 438 & $8^{\circ} 37^{\prime} 12.3^{\prime \prime}$ & $47^{\circ} 41^{\prime} 23.2^{\prime \prime}$ & -0.62 & 09:50 \\
\hline SIA & Segl-Maria & 1804 & $9^{\circ} 45^{\prime} 44.4^{\prime \prime}$ & $46^{\circ} 25^{\prime} 56.4^{\prime \prime}$ & -1.93 & 09:30 \\
\hline SIO & Sion & 482 & $7^{\circ} 19^{\prime} 48.6^{\prime \prime}$ & $46^{\circ} 13^{\prime} 06.9^{\prime \prime}$ & -1.57 & 09:40 \\
\hline SMA & Zürich / Fluntern & 555 & $8^{\circ} 33^{\prime} 56.7^{\prime \prime}$ & $47^{\circ} 22^{\prime} 40.4^{\prime \prime}$ & -0.07 & $10: 10$ \\
\hline SMM & Sta. Maria, Val Müstair & 1383 & $10^{\circ} 25^{\prime} 34.7^{\prime \prime}$ & $46^{\circ} 36^{\prime} 08.1^{\prime \prime}$ & -1.61 & $10: 00$ \\
\hline SPF & Schüpfheim & 742 & $8^{\circ} 00^{\prime} 44.8^{\prime \prime}$ & $46^{\circ} 56^{\prime} 49.3^{\prime \prime}$ & -1.72 & 09:50 \\
\hline SRS & Schiers & 626 & $9^{\circ} 40^{\prime} 05.0^{\prime \prime}$ & $46^{\circ} 58^{\prime} 31.8^{\prime \prime}$ & -2.00 & 09:50 \\
\hline STG & St. Gallen & 775 & $9^{\circ} 23^{\prime} 54.5^{\prime \prime}$ & $47^{\circ} 25^{\prime} 31.6^{\prime \prime}$ & -1.49 & $10: 10$ \\
\hline STP & Stöckalp & 1070 & $8^{\circ} 16^{\prime} 52.3^{\prime \prime}$ & $46^{\circ} 48^{\prime} 00.0^{\prime \prime}$ & -1.62 & $10: 00$ \\
\hline TAE & Aadorf / Tänikon & 539 & $8^{\circ} 54^{\prime} 17.6^{\prime \prime}$ & $47^{\circ} 28^{\prime} 47.5^{\prime \prime}$ & -0.61 & $10: 00$ \\
\hline THU & Thun & 570 & $7^{\circ} 35^{\prime} 06.8^{\prime \prime}$ & $46^{\circ} 44^{\prime} 59.5^{\prime \prime}$ & -0.42 & 09:50 \\
\hline TIT & Titlis & 3040 & $8^{\circ} 25^{\prime} 32.9^{\prime \prime}$ & $46^{\circ} 46^{\prime} 13.8^{\prime \prime}$ & -1.57 & $10: 20$ \\
\hline TSG & Arosa / Tschuggen & 1840 & $9^{\circ} 39^{\prime} 58.8^{\prime \prime}$ & $46^{\circ} 46^{\prime} 51.4^{\prime \prime}$ & -5.44 & 09:40 \\
\hline ULR & Ulrichen & 1345 & $8^{\circ} 18^{\prime} 29.3^{\prime \prime}$ & $46^{\circ} 30^{\prime} 17.4^{\prime \prime}$ & 0.25 & 08:40 \\
\hline VAB & Valbella & 1569 & $9^{\circ} 33^{\prime} 15.9^{\prime \prime}$ & $46^{\circ} 45^{\prime} 18.1^{\prime \prime}$ & -0.77 & $10: 00$ \\
\hline VAD & Vaduz & 457 & $9^{\circ} 31^{\prime} 03.0^{\prime \prime}$ & $47^{\circ} 07^{\prime} 38.8^{\prime \prime}$ & 0.15 & 09:50 \\
\hline VIO & Vicosoprano & 1089 & $9^{\circ} 37^{\prime} 40.1^{\prime \prime}$ & $46^{\circ} 21^{\prime} 10.9^{\prime \prime}$ & -1.68 & $10: 10$ \\
\hline VIS & Visp & 639 & $7^{\circ} 50^{\prime} 34.6^{\prime \prime}$ & $46^{\circ} 18^{\prime} 10.3^{\prime \prime}$ & -0.76 & 09:50 \\
\hline VIT & Villars-Tiercelin & 859 & $6^{\circ} 42^{\prime} 36.3^{\prime \prime}$ & $46^{\circ} 37^{\prime} 18.4^{\prime \prime}$ & -1.46 & 09:50 \\
\hline VSBLI & Blinnen & 1530 & $8^{\circ} 15^{\prime} 35.3^{\prime \prime}$ & $46^{\circ} 27^{\prime} 25.2^{\prime \prime}$ & 0.01 & $10: 10$ \\
\hline VSBRU & Bruchji & 2300 & $7^{\circ} 58^{\prime} 19.9^{\prime \prime}$ & $46^{\circ} 22^{\prime} 43.0^{\prime \prime}$ & -3.60 & 09:10 \\
\hline VSBSP & Bourg-St-Pierre & 1826 & $7^{\circ} 11^{\prime} 53.6^{\prime \prime}$ & $45^{\circ} 55^{\prime} 39.3^{\prime \prime}$ & -3.09 & $10: 10$ \\
\hline VSCHO & Choëx & 896 & $6^{\circ} 57^{\prime} 40.9^{\prime \prime}$ & $46^{\circ} 13^{\prime} 55.7^{\prime \prime}$ & -2.09 & 09:40 \\
\hline VSCOL & Les Collons & 1787 & $7^{\circ} 23^{\prime} 14.5^{\prime \prime}$ & $46^{\circ} 10^{\prime} 44.3^{\prime \prime}$ & -0.51 & 09:50 \\
\hline VSDER & Derborence & 1380 & $7^{\circ} 13^{\prime} 57.0^{\prime \prime}$ & $46^{\circ} 17^{\prime} 18.6^{\prime \prime}$ & -4.14 & 09:40 \\
\hline VSDUR & Durnand & 2000 & $7^{\circ} 05^{\prime} 02.7^{\prime \prime}$ & $46^{\circ} 03^{\prime} 06.4^{\prime \prime}$ & -2.38 & $09: 50$ \\
\hline VSERG & Ergisch & 1133 & $7^{\circ} 42^{\prime} 58.7^{\prime \prime}$ & $46^{\circ} 17^{\prime} 34.8^{\prime \prime}$ & -1.51 & 09:40 \\
\hline VSISE & Isérables & 1237 & $7^{\circ} 14^{\prime} 55.5^{\prime \prime}$ & $46^{\circ} 09^{\prime} 39.3^{\prime \prime}$ & -1.00 & 08:40 \\
\hline VSNEN & Nendaz & 1938 & $7^{\circ} 18^{\prime} 41.1^{\prime \prime}$ & $46^{\circ} 07^{\prime} 19.5^{\prime \prime}$ & -3.19 & 09:40 \\
\hline VSSIE & Sierre & 535 & $7^{\circ} 33^{\prime} 23.0^{\prime \prime}$ & $46^{\circ} 17^{\prime} 55.6^{\prime \prime}$ & -3.56 & 09:50 \\
\hline VSSOR & Sorniot-Lac Inférieur & 2005 & $7^{\circ} 06^{\prime} 00.2^{\prime \prime}$ & $46^{\circ} 10^{\prime} 01.0^{\prime \prime}$ & -5.78 & 09:50 \\
\hline VSTRI & Trient & 1290 & $6^{\circ} 59^{\prime} 41.6^{\prime \prime}$ & $46^{\circ} 3^{\prime} 30.2^{\prime \prime} 0$ & -1.97 & 09:30 \\
\hline VSTSN & Tsanfleuron & 2052 & $7^{\circ} 18^{\prime} 06.5^{\prime \prime}$ & $46^{\circ} 19^{\prime} 14.5^{\prime \prime}$ & -5.43 & $10: 00$ \\
\hline VSVIS & Visperterminen & 1360 & $7^{\circ} 54^{\prime} 15.1^{\prime \prime}$ & $46^{\circ} 15^{\prime} 46.4^{\prime \prime}$ & -0.71 & 09:50 \\
\hline WAE & Wädenswil & 485 & $8^{\circ} 40^{\prime} 39.8^{\prime \prime}$ & $47^{\circ} 13^{\prime} 14.3^{\prime \prime}$ & -1.55 & $10: 20$ \\
\hline WFJ & Weissfluhjoch & 2691 & $9^{\circ} 48^{\prime} 23.0^{\prime \prime}$ & $46^{\circ} 49^{\prime} 60.0^{\prime \prime}$ & -1.22 & $10: 00$ \\
\hline
\end{tabular}


Table S2. (continued)

\begin{tabular}{|c|c|c|c|c|c|c|}
\hline CODE & Station Name & Elevation $(\mathrm{m})$ & Longitude (E) & Latitude (N) & $\Delta T(\mathrm{~K})$ & $t(\Delta T)$ \\
\hline WSLALB & Alptal Bestand & 1168 & $8^{\circ} 42^{\prime} 47.6^{\prime \prime}$ & $47^{\circ} 02^{\prime} 55.8^{\prime \prime}$ & -1.33 & $10: 10$ \\
\hline WSLBAB & Beatenberg Bestand & 1522 & $7^{\circ} 45^{\prime} 48.6^{\prime \prime}$ & $46^{\circ} 42^{\prime} 00.4^{\prime \prime}$ & -1.65 & $10: 10$ \\
\hline WSLBAF & Beatenberg Freiland & 1560 & $7^{\circ} 46^{\prime} 18.2^{\prime \prime}$ & $46^{\circ} 42^{\prime} 02.1^{\prime \prime}$ & -1.94 & 09:50 \\
\hline WSLBTB & Bettlachstock Bestand & 1050 & $7^{\circ} 24^{\prime} 38.1^{\prime \prime}$ & $47^{\circ} 13^{\prime} 20.2^{\prime \prime}$ & -3.10 & 10:00 \\
\hline WSLBTF & Bettlachstock Freiland & 1100 & $7^{\circ} 25^{\prime} 02.8^{\prime \prime}$ & $47^{\circ} 13^{\prime} 28.2^{\prime \prime}$ & -4.71 & 10:00 \\
\hline WSLCIB & Chironico Bestand & 1460 & $8^{\circ} 49^{\prime} 06.1^{\prime \prime}$ & $46^{\circ} 26^{\prime} 28.3^{\prime \prime}$ & -0.40 & 10:10 \\
\hline WSLCIF & Chironico Freiland & 1380 & $8^{\circ} 48^{\prime} 46.9^{\prime \prime}$ & $46^{\circ} 26^{\prime} 47.0^{\prime \prime}$ & -1.81 & 09:50 \\
\hline WSLCLB & Celerina Bestand & 1760 & $9^{\circ} 52^{\prime} 33.1^{\prime \prime}$ & $46^{\circ} 30^{\prime} 21.9^{\prime \prime}$ & -1.67 & 10:00 \\
\hline WSLCLF & Celerina Freiland & 1865 & $9^{\circ} 53^{\prime} 18.2^{\prime \prime}$ & $46^{\circ} 29^{\prime} 33.1^{\prime \prime}$ & -3.70 & 09:50 \\
\hline WSLISB & Isone Bestand & 1194 & $9^{\circ} 00^{\prime} 28.8^{\prime \prime}$ & $46^{\circ} 07^{\prime} 30.4^{\prime \prime}$ & -0.57 & $10: 30$ \\
\hline WSLISF & Isone Freiland & 1149 & $9^{\circ} 00^{\prime} 25.1^{\prime \prime}$ & $46^{\circ} 07^{\prime} 34.4^{\prime \prime}$ & -1.10 & $10: 30$ \\
\hline WSLJUB & Jussy Bestand & 500 & $6^{\circ} 17^{\prime} 35.3^{\prime \prime}$ & $46^{\circ} 13^{\prime} 52.7^{\prime \prime}$ & -2.33 & 10:00 \\
\hline WSLJUF & Jussy Freiland & 497 & $6^{\circ} 17^{\prime} 12.6^{\prime \prime}$ & $46^{\circ} 13^{\prime} 43.1^{\prime \prime}$ & -4.09 & 09:50 \\
\hline WSLLAB & Lausanne Bestand & 806 & $6^{\circ} 39^{\prime} 29.1^{\prime \prime}$ & $46^{\circ} 35^{\prime} 01.4^{\prime \prime}$ & -1.66 & 10:00 \\
\hline WSLLAF & Lausanne Freiland & 790 & $6^{\circ} 38^{\prime} 56.4^{\prime \prime}$ & $46^{\circ} 33^{\prime} 33.4^{\prime \prime}$ & -3.44 & 09:50 \\
\hline WSLLEB & Lens Bestand & 1069 & $7^{\circ} 26^{\prime} 09.1^{\prime \prime}$ & $46^{\circ} 16^{\prime} 06.9^{\prime \prime}$ & -0.82 & $10: 10$ \\
\hline WSLNAB & Nationalpark Bestand & 1913 & $10^{\circ} 14^{\prime} 28.0^{\prime \prime}$ & $46^{\circ} 39^{\prime} 33.1^{\prime \prime}$ & -2.21 & 10:00 \\
\hline WSLNAF & Nationalpark Freiland & 1899 & $10^{\circ} 13^{\prime} 47.8^{\prime \prime}$ & $46^{\circ} 39^{\prime} 44.5^{\prime \prime}$ & -1.55 & $10: 00$ \\
\hline WSLNEB & Neunkirch Bestand & 561 & $8^{\circ} 32^{\prime} 05.1^{\prime \prime}$ & $47^{\circ} 41^{\prime} 02.3^{\prime \prime}$ & -0.96 & $10: 20$ \\
\hline WSLNEF & Neunkrich Freiland & 463 & $8^{\circ} 31^{\prime} 34.8^{\prime \prime}$ & $47^{\circ} 41^{\prime} 23.3^{\prime \prime}$ & -3.57 & $10: 00$ \\
\hline WSLNOB & Novaggio Bestand & 928 & $8^{\circ} 50^{\prime} 04.0^{\prime \prime}$ & $46^{\circ} 01^{\prime} 19.5^{\prime \prime}$ & -0.99 & 09:40 \\
\hline WSLNOF & Novaggio Freiland & 1055 & $8^{\circ} 50^{\prime} 04.7^{\prime \prime}$ & $46^{\circ} 1^{\prime} 28.8^{\prime \prime} 0$ & -0.97 & $09: 30$ \\
\hline WSLOTB & Othmarsingen Bestand & 474 & $8^{\circ} 13^{\prime} 37.3^{\prime \prime}$ & $47^{\circ} 23^{\prime} 55.2^{\prime \prime}$ & -2.79 & $10: 00$ \\
\hline WSLOTF & Othmarsingen Freiland & 461 & $8^{\circ} 13^{\prime} 23.1^{\prime \prime}$ & $47^{\circ} 24^{\prime} 00.8^{\prime \prime}$ & -3.41 & $10: 00$ \\
\hline WSLSCB & Schänis Bestand & 709 & $9^{\circ} 04^{\prime} 00.3^{\prime \prime}$ & $47^{\circ} 09^{\prime} 56.3^{\prime \prime}$ & -0.52 & $10: 20$ \\
\hline WSLSCF & Schänis Freiland & 626 & $9^{\circ} 03^{\prime} 34.4^{\prime \prime}$ & $47^{\circ} 09^{\prime} 04.4^{\prime \prime}$ & -2.15 & $10: 10$ \\
\hline WSLVOB & Vordemwald Bestand & 482 & $7^{\circ} 53^{\prime} 13.4^{\prime \prime}$ & $47^{\circ} 16^{\prime} 26.0^{\prime \prime}$ & -0.93 & $10: 10$ \\
\hline WSLVOF & Vordemwald Freiland & 480 & $7^{\circ} 54^{\prime} 39.8^{\prime \prime}$ & $47^{\circ} 16^{\prime} 08.4^{\prime \prime}$ & -2.02 & 09:50 \\
\hline WSLVSB & Visp Bestand & 698 & $7^{\circ} 51^{\prime} 26.3^{\prime \prime}$ & $46^{\circ} 17^{\prime} 48.9^{\prime \prime}$ & -2.46 & $10: 00$ \\
\hline WSLVSF & Visp Freiland & 640 & $7^{\circ} 50^{\prime} 33.4^{\prime \prime}$ & $46^{\circ} 18^{\prime} 09.7^{\prime \prime}$ & -1.43 & 09:50 \\
\hline WYN & Wynau & 422 & $7^{\circ} 47^{\prime} 14.7^{\prime \prime}$ & $47^{\circ} 15^{\prime} 18.1^{\prime \prime}$ & -1.07 & 09:50 \\
\hline ZER & Zermatt & 1638 & $7^{\circ} 45^{\prime} 11.2^{\prime \prime}$ & $46^{\circ} 01^{\prime} 44.9^{\prime \prime}$ & -2.55 & 09:10 \\
\hline
\end{tabular}


Table S3. MeteoSwiss standard weather stations measured wind speed and wind direction and their classification according to topography. Where possible, the orientation of the valley and the slope angle are given.

\begin{tabular}{|c|c|c|c|c|c|c|c|}
\hline Code & Location & Longitude & Latitude & Elevation $(\mathrm{m})$ & Classification & Up-Valley Dir. & Slope Angle \\
\hline $\mathrm{ABO}$ & Adelboden & $7^{\circ} 33^{\prime} 37.5^{\prime \prime} \mathrm{E}$ & $46^{\circ} 29^{\prime} 32.1^{\prime \prime} \mathrm{N}$ & 1326 & valley & $225^{\circ}$ & $310^{\circ}$ \\
\hline AEG & Oberägeri & $8^{\circ} 36^{\prime} 29.1^{\prime \prime} \mathrm{E}$ & $47^{\circ} 08^{\prime} 00.9^{\prime \prime} \mathrm{N}$ & 724 & flat & $315^{\circ}$ & $45^{\circ}$ \\
\hline AIG & Aigle & $6^{\circ} 55^{\prime} 27.9^{\prime \prime} \mathrm{E}$ & $46^{\circ} 19^{\prime} 35.9^{\prime \prime} \mathrm{N}$ & 381 & valley & $315^{\circ}$ & \\
\hline ALP & Alpnach & $8^{\circ} 17^{\prime} 08.6^{\prime \prime} \mathrm{E}$ & $46^{\circ} 56^{\prime} 34.8^{\prime \prime} \mathrm{N}$ & 436 & valley & $200^{\circ}$ & \\
\hline ALT & Altdorf & $8^{\circ} 37^{\prime} 18.5^{\prime \prime} \mathrm{E}$ & $46^{\circ} 53^{\prime} 13.3^{\prime \prime} \mathrm{N}$ & 438 & valley & $150^{\circ}$ & \\
\hline AND & Andeer & $9^{\circ} 25^{\prime} 54.9^{\prime \prime} \mathrm{E}$ & $46^{\circ} 36^{\prime} 36.4^{\prime \prime} \mathrm{N}$ & 987 & valley & $200^{\circ}$ & $110^{\circ}$ \\
\hline ANT & Andermatt & $8^{\circ} 34^{\prime} 50.0^{\prime \prime} \mathrm{E}$ & $46^{\circ} 37^{\prime} 51.3^{\prime \prime} \mathrm{N}$ & 1438 & valley & $225^{\circ}$ & \\
\hline ARD & Arosa & $9^{\circ} 40^{\prime} 28.3^{\prime \prime} \mathrm{E}$ & $46^{\circ} 46^{\prime} 45.0^{\prime \prime} \mathrm{N}$ & 1840 & slope & $255^{\circ}$ & $310^{\circ}$ \\
\hline ARH & Altenrhein & $9^{\circ} 34^{\prime} 00.5^{\prime \prime} \mathrm{E}$ & $47^{\circ} 29^{\prime} 01.2^{\prime \prime} \mathrm{N}$ & 398 & flat & $120^{\circ}$ & $190^{\circ}$ \\
\hline ATT & Les Attelas & $7^{\circ} 16^{\prime} 07.5^{\prime \prime} \mathrm{E}$ & $46^{\circ} 05^{\prime} 56.8^{\prime \prime} \mathrm{N}$ & 2730 & mtohill & $160^{\circ}$ & \\
\hline BAN & Bantiger & $7^{\circ} 31^{\prime} 43.2^{\prime \prime} \mathrm{E}$ & $46^{\circ} 58^{\prime} 40.1^{\prime \prime} \mathrm{N}$ & 941 & mtohill & $135^{\circ}$ & \\
\hline BAS & Basel / Binningen & $7^{\circ} 35^{\prime} 00.8^{\prime \prime} \mathrm{E}$ & $47^{\circ} 32^{\prime} 27.7^{\prime \prime} \mathrm{N}$ & 316 & slope & $110^{\circ}$ & $190^{\circ}$ \\
\hline $\mathrm{BEH}$ & Passo del Bernina & $10^{\circ} 01^{\prime} 10.5^{\prime \prime} \mathrm{E}$ & $46^{\circ} 24^{\prime} 33.0^{\prime \prime} \mathrm{N}$ & 2260 & slope & $135^{\circ}$ & $45^{\circ}$ \\
\hline BER & Bern / Zollikofen & $7^{\circ} 27^{\prime} 50.4^{\prime \prime} \mathrm{E}$ & $46^{\circ} 59^{\prime} 26.7^{\prime \prime} \mathrm{N}$ & 552 & flat & $0^{\circ}$ & \\
\hline BEZ & Beznau & $8^{\circ} 13^{\prime} 59.8^{\prime \prime} \mathrm{E}$ & $47^{\circ} 33^{\prime} 26.0^{\prime \prime} \mathrm{N}$ & 325 & valley & $255^{\circ}$ & \\
\hline BHF & Oberrütti / Büelhof & $7^{\circ} 16^{\prime} 58.7^{\prime \prime} \mathrm{E}$ & $46^{\circ} 57^{\prime} 07.6^{\prime \prime} \mathrm{N}$ & 775 & mtohill & $310^{\circ}$ & \\
\hline BIE & Bière & $6^{\circ} 20^{\prime} 32.6^{\prime \prime} \mathrm{E}$ & $46^{\circ} 31^{\prime} 29.5^{\prime \prime} \mathrm{N}$ & 683 & flat & $225^{\circ}$ & $315^{\circ}$ \\
\hline BIN & Binn & $8^{\circ} 11^{\prime} 27.3^{\prime \prime} \mathrm{E}$ & $46^{\circ} 21^{\prime} 55.7^{\prime \prime} \mathrm{N}$ & 1448 & valley & $75^{\circ}$ & $170^{\circ}$ \\
\hline BIZ & Bischofszell & $9^{\circ} 14^{\prime} 05.0^{\prime \prime} \mathrm{E}$ & $47^{\circ} 29^{\prime} 50.6^{\prime \prime} \mathrm{N}$ & 470 & flat & $60^{\circ}$ & \\
\hline BLA & Blatten, Lötschental & $7^{\circ} 49^{\prime} 23.5^{\prime \prime} \mathrm{E}$ & $46^{\circ} 25^{\prime} 13.6^{\prime \prime} \mathrm{N}$ & 1538 & valley & $50^{\circ}$ & $100^{\circ}$ \\
\hline BOL & Boltigen & $7^{\circ} 23^{\prime} 03.1^{\prime \prime} \mathrm{E}$ & $46^{\circ} 37^{\prime} 24.7^{\prime \prime} \mathrm{N}$ & 820 & valley & $200^{\circ}$ & \\
\hline BOU & Bouveret & $6^{\circ} 51^{\prime} 25.2^{\prime \prime} \mathrm{E}$ & $46^{\circ} 23^{\prime} 36.4^{\prime \prime} \mathrm{N}$ & 374 & flat & $135^{\circ}$ & \\
\hline BRL & La Brévine & $6^{\circ} 36^{\prime} 37.0^{\prime \prime} \mathrm{E}$ & $46^{\circ} 59^{\prime} 01.8^{\prime \prime} \mathrm{N}$ & 1050 & valley & $270^{\circ}$ & \\
\hline BRZ & Brienz & $8^{\circ} 03^{\prime} 39.1^{\prime \prime} \mathrm{E}$ & $46^{\circ} 44^{\prime} 26.6^{\prime \prime} \mathrm{N}$ & 567 & valley & $85^{\circ}$ & \\
\hline BUF & Buffalora & $10^{\circ} 16^{\prime} 01.9^{\prime \prime} \mathrm{E}$ & $46^{\circ} 38^{\prime} 53.3^{\prime \prime} \mathrm{N}$ & 1968 & slope & $170^{\circ}$ & $45^{\circ}$ \\
\hline BUS & Buchs / Aarau & $8^{\circ} 04^{\prime} 46.0^{\prime \prime} \mathrm{E}$ & $47^{\circ} 23^{\prime} 03.6^{\prime \prime} \mathrm{N}$ & 386 & flat & $170^{\circ}$ & \\
\hline $\mathrm{CDF}$ & La Chaux-de-Fonds & $6^{\circ} 47^{\prime} 32.3^{\prime \prime} \mathrm{E}$ & $47^{\circ} 04^{\prime} 58.6^{\prime \prime} \mathrm{N}$ & 1018 & valley & $45^{\circ}$ & $315^{\circ}$ \\
\hline CEV & Cevio & $8^{\circ} 36^{\prime} 11.4^{\prime \prime} \mathrm{E}$ & $46^{\circ} 19^{\prime} 13.8^{\prime \prime} \mathrm{N}$ & 417 & valley & $0^{\circ}$ & \\
\hline CGI & Nyon / Changins & $6^{\circ} 13^{\prime} 39.9^{\prime \prime} \mathrm{E}$ & $46^{\circ} 24^{\prime} 03.7^{\prime \prime} \mathrm{N}$ & 455 & mtohill & $30^{\circ}$ & \\
\hline CHA & Chasseral & $7^{\circ} 03^{\prime} 15.8^{\prime \prime} \mathrm{E}$ & $47^{\circ} 07^{\prime} 54.4^{\prime \prime} \mathrm{N}$ & 1599 & mtohill & $240^{\circ}$ & \\
\hline CHB & Les Charbonnières & $6^{\circ} 18^{\prime} 44.7^{\prime \prime} \mathrm{E}$ & $46^{\circ} 40^{\prime} 12.5^{\prime \prime} \mathrm{N}$ & 1045 & slope & $225^{\circ}$ & $315^{\circ}$ \\
\hline CHD & Château-d'Oex & $7^{\circ} 08^{\prime} 22.7^{\prime \prime} \mathrm{E}$ & $46^{\circ} 28^{\prime} 47.3^{\prime \prime} \mathrm{N}$ & 1029 & slope & $100^{\circ}$ & $0^{\circ}$ \\
\hline CHM & Chaumont & $6^{\circ} 58^{\prime} 43.7^{\prime \prime} \mathrm{E}$ & $47^{\circ} 02^{\prime} 57.0^{\prime \prime} \mathrm{N}$ & 1136 & mtohill & $200^{\circ}$ & \\
\hline $\mathrm{CHU}$ & Chur & $9^{\circ} 31^{\prime} 50.1^{\prime \prime} \mathrm{E}$ & $46^{\circ} 52^{\prime} 13.5^{\prime \prime} \mathrm{N}$ & 556 & valley & $200^{\circ}$ & \\
\hline CHZ & Cham & $8^{\circ} 27^{\prime} 52.7^{\prime \prime} \mathrm{E}$ & $47^{\circ} 11^{\prime} 17.8^{\prime \prime} \mathrm{N}$ & 442 & mtohill & $170^{\circ}$ & \\
\hline CIM & Cimetta & $8^{\circ} 47^{\prime} 29.9^{\prime \prime} \mathrm{E}$ & $46^{\circ} 12^{\prime} 01.5^{\prime \prime} \mathrm{N}$ & 1661 & mtohill & $345^{\circ}$ & \\
\hline CMA & Crap Masegn & $9^{\circ} 10^{\prime} 48.0^{\prime \prime} \mathrm{E}$ & $46^{\circ} 50^{\prime} 32.3^{\prime \prime} \mathrm{N}$ & 2480 & mtohill & $320^{\circ}$ & \\
\hline $\mathrm{COM}$ & Acquarossa / Comprovasco & $8^{\circ} 56^{\prime} 08.4^{\prime \prime} \mathrm{E}$ & $46^{\circ} 27^{\prime} 33.9^{\prime \prime} \mathrm{N}$ & 575 & valley & $350^{\circ}$ & $270^{\circ}$ \\
\hline $\mathrm{COV}$ & Piz Corvatsch & $9^{\circ} 49^{\prime} 16.5^{\prime \prime} \mathrm{E}$ & $46^{\circ} 25^{\prime} 04.8^{\prime \prime} \mathrm{N}$ & 3302 & mtohill & $45^{\circ}$ & \\
\hline $\mathrm{COY}$ & Courtelary & $7^{\circ} 05^{\prime} 26.3^{\prime \prime} \mathrm{E}$ & $47^{\circ} 10^{\prime} 50.9^{\prime \prime} \mathrm{N}$ & 695 & slope & $250^{\circ}$ & $170^{\circ}$ \\
\hline CRM & Cressier & $7^{\circ} 03^{\prime} 32.8^{\prime \prime} \mathrm{E}$ & $47^{\circ} 02^{\prime} 51.4^{\prime \prime} \mathrm{N}$ & 431 & flat & $250^{\circ}$ & \\
\hline DAV & Davos & $9^{\circ} 50^{\prime} 36.6^{\prime \prime} \mathrm{E}$ & $46^{\circ} 48^{\prime} 46.6^{\prime \prime} \mathrm{N}$ & 1594 & slope & $220^{\circ}$ & $310^{\circ}$ \\
\hline DEM & Delémont & $7^{\circ} 20^{\prime} 58.4^{\prime \prime} \mathrm{E}$ & $47^{\circ} 21^{\prime} 06.1^{\prime \prime} \mathrm{N}$ & 439 & slope & & $160^{\circ}$ \\
\hline DIA & Les Diablerets & $7^{\circ} 12^{\prime} 13.6^{\prime \prime} \mathrm{E}$ & $46^{\circ} 19^{\prime} 36.3^{\prime \prime} \mathrm{N}$ & 2964 & mtohill & $325^{\circ}$ & \\
\hline DIS & Disentis / Sedrun & $8^{\circ} 51^{\prime} 12.4^{\prime \prime} \mathrm{E}$ & $46^{\circ} 42^{\prime} 23.5^{\prime \prime} \mathrm{N}$ & 1197 & slope & $225^{\circ}$ & $315^{\circ}$ \\
\hline DOL & La Dôle & $6^{\circ} 05^{\prime} 58.1^{\prime \prime} \mathrm{E}$ & $46^{\circ} 25^{\prime} 28.9^{\prime \prime} \mathrm{N}$ & 1669 & mtohill & $225^{\circ}$ & \\
\hline EBK & Ebnat-Kappel & $9^{\circ} 06^{\prime} 30.5^{\prime \prime} \mathrm{E}$ & $47^{\circ} 16^{\prime} 24.2^{\prime \prime} \mathrm{N}$ & 623 & valley & $140^{\circ}$ & \\
\hline EGH & Eggishorn & $8^{\circ} 05^{\prime} 33.8^{\prime \prime} \mathrm{E}$ & $46^{\circ} 25^{\prime} 35.5^{\prime \prime} \mathrm{N}$ & 2893 & mtohill & $20^{\circ}$ & \\
\hline EGO & Egolzwil & $8^{\circ} 00^{\prime} 17.1^{\prime \prime} \mathrm{E}$ & $47^{\circ} 10^{\prime} 45.9^{\prime \prime} \mathrm{N}$ & 521 & mtohill & $20^{\circ}$ & \\
\hline EIN & Einsiedeln & $8^{\circ} 45^{\prime} 23.6^{\prime \prime} \mathrm{E}$ & $47^{\circ} 07^{\prime} 58.9^{\prime \prime} \mathrm{N}$ & 910 & slope & $170^{\circ}$ & $313^{\circ}$ \\
\hline ELM & Elm & $9^{\circ} 10^{\prime} 31.3^{\prime \prime} \mathrm{E}$ & $46^{\circ} 55^{\prime} 25.5^{\prime \prime} \mathrm{N}$ & 958 & slope & $190^{\circ}$ & $120^{\circ}$ \\
\hline EMM & Emmen & $8^{\circ} 17^{\prime} 45.5^{\prime \prime} \mathrm{E}$ & $47^{\circ} 05^{\prime} 03.5^{\prime \prime} \mathrm{N}$ & 426 & flat & $225^{\circ}$ & \\
\hline ENG & Engelberg & $8^{\circ} 24^{\prime} 37.6^{\prime \prime} \mathrm{E}$ & $46^{\circ} 49^{\prime} 18.8^{\prime \prime} \mathrm{N}$ & 1035 & slope & $30^{\circ}$ & $120^{\circ}$ \\
\hline EVI & Evionnaz & $7^{\circ} 01^{\prime} 36.3^{\prime \prime} \mathrm{E}$ & $46^{\circ} 10^{\prime} 58.6^{\prime \prime} \mathrm{N}$ & 482 & slope & 280 & $0^{\circ}$ \\
\hline EVO & Evolène / Villa & $7^{\circ} 30^{\prime} 31.2^{\prime \prime} \mathrm{E}$ & $46^{\circ} 06^{\prime} 43.7^{\prime \prime} \mathrm{N}$ & 1825 & slope & $40^{\circ}$ & $130^{\circ}$ \\
\hline
\end{tabular}


Table S3. (continued)

\begin{tabular}{|c|c|c|c|c|c|c|c|}
\hline Code & Location & Longitude & Latitude & Elevation (m) & Classification & Valley Dir. & Slope Angle \\
\hline FAH & Fahy & $6^{\circ} 56^{\prime} 27.9^{\prime \prime} \mathrm{E}$ & $47^{\circ} 25^{\prime} 25.7^{\prime \prime} \mathrm{N}$ & 596 & mtohill & $260^{\circ}$ & $170^{\circ}$ \\
\hline FRE & Bullet / La Frétaz & $6^{\circ} 34^{\prime} 34.7^{\prime \prime} \mathrm{E}$ & $46^{\circ} 50^{\prime} 26.2^{\prime \prime} \mathrm{N}$ & 1205 & mtohill & $225^{\circ}$ & $315^{\circ}$ \\
\hline GEN & Monte Generoso & $9^{\circ} 01^{\prime} 04.3^{\prime \prime} \mathrm{E}$ & $45^{\circ} 55^{\prime} 39.3^{\prime \prime} \mathrm{N}$ & 1600 & mtohill & $45^{\circ}$ & \\
\hline GES & Gersau & $8^{\circ} 31^{\prime} 24.4^{\prime \prime} \mathrm{E}$ & $46^{\circ} 59^{\prime} 45.8^{\prime \prime} \mathrm{N}$ & 519 & slope & $60^{\circ}$ & $330^{\circ}$ \\
\hline GIH & Giswil & $8^{\circ} 11^{\prime} 24.7^{\prime \prime} \mathrm{E}$ & $46^{\circ} 50^{\prime} 58.2^{\prime \prime} \mathrm{N}$ & 471 & flat & $200^{\circ}$ & \\
\hline GLA & Glarus & $9^{\circ} 04^{\prime} 00.9^{\prime \prime} \mathrm{E}$ & $47^{\circ} 02^{\prime} 04.5^{\prime \prime} \mathrm{N}$ & 516 & slope & $135^{\circ}$ & $200^{\circ}$ \\
\hline GOE & Gösgen & $7^{\circ} 58^{\prime} 25.2^{\prime \prime} \mathrm{E}$ & $47^{\circ} 21^{\prime} 46.9^{\prime \prime} \mathrm{N}$ & 380 & flat & $290^{\circ}$ & \\
\hline GOR & Gornergrat & $7^{\circ} 47^{\prime} 08.7^{\prime \prime} \mathrm{E}$ & $45^{\circ} 59^{\prime} 01.1^{\prime \prime} \mathrm{N}$ & 3129 & mtohill & $270^{\circ}$ & \\
\hline GRA & Fribourg / Posieux & $7^{\circ} 06^{\prime} 49.3^{\prime \prime} \mathrm{E}$ & $46^{\circ} 46^{\prime} 17.0^{\prime \prime} \mathrm{N}$ & 646 & slope & $225^{\circ}$ & $315^{\circ}$ \\
\hline GRE & Grenchen & $7^{\circ} 24^{\prime} 54.4^{\prime \prime} \mathrm{E}$ & $47^{\circ} 10^{\prime} 44.7^{\prime \prime} \mathrm{N}$ & 430 & flat & $245^{\circ}$ & \\
\hline GRH & Grimsel Hospiz & $8^{\circ} 19^{\prime} 59.7^{\prime \prime} \mathrm{E}$ & $46^{\circ} 34^{\prime} 18.1^{\prime \prime} \mathrm{N}$ & 1980 & mtohill & $225^{\circ}$ & \\
\hline GRO & Grono & $9^{\circ} 09^{\prime} 49.5^{\prime \prime} \mathrm{E}$ & $46^{\circ} 15^{\prime} 18.3^{\prime \prime} \mathrm{N}$ & 323 & valley & $30^{\circ}$ & \\
\hline GSB & Col du Grand St-Bernard & $7^{\circ} 10^{\prime} 14.9^{\prime \prime} \mathrm{E}$ & $45^{\circ} 52^{\prime} 07.6^{\prime \prime} \mathrm{N}$ & 2472 & valley & $270^{\circ}$ & $0^{\circ}$ \\
\hline GUB & Regensdorf / Gubrist & $8^{\circ} 28^{\prime} 44.1^{\prime \prime} \mathrm{E}$ & $47^{\circ} 29^{\prime} 59.5^{\prime \prime} \mathrm{N}$ & 410 & mtohill & $270^{\circ}$ & \\
\hline GUE & Gütsch ob Andermatt & $8^{\circ} 36^{\prime} 54.2^{\prime \prime} \mathrm{E}$ & $46^{\circ} 39^{\prime} 08.8^{\prime \prime} \mathrm{N}$ & 2283 & mtohill & $250^{\circ}$ & $0^{\circ}$ \\
\hline GUT & Güttingen & $9^{\circ} 16^{\prime} 45.8^{\prime \prime} \mathrm{E}$ & $47^{\circ} 36^{\prime} 06.1^{\prime \prime} \mathrm{N}$ & 440 & slope & $11^{\circ} 0$ & $200^{\circ}$ \\
\hline GVE & Genève-Cointrin & $6^{\circ} 07^{\prime} 39.9^{\prime \prime} \mathrm{E}$ & $46^{\circ} 14^{\prime} 51.0^{\prime \prime} \mathrm{N}$ & 412 & flat & $45^{\circ}$ & \\
\hline HAI & Salen-Reutenen & $9^{\circ} 01^{\prime} 26.1^{\prime \prime} \mathrm{E}$ & $47^{\circ} 39^{\prime} 04.4^{\prime \prime} \mathrm{N}$ & 718 & flat & $270^{\circ}$ & \\
\hline HLL & Hallau & $8^{\circ} 28^{\prime} 13.7^{\prime \prime} \mathrm{E}$ & $47^{\circ} 41^{\prime} 50.2^{\prime \prime} \mathrm{N}$ & 419 & valley & $40^{\circ}$ & $310^{\circ}$ \\
\hline HOE & Hörnli & $8^{\circ} 56^{\prime} 29.9^{\prime \prime} \mathrm{E}$ & $47^{\circ} 22^{\prime} 15.1^{\prime \prime} \mathrm{N}$ & 1144 & mtohill & $350^{\circ}$ & \\
\hline INT & Interlaken & $7^{\circ} 52^{\prime} 12.5^{\prime \prime} \mathrm{E}$ & $46^{\circ} 40^{\prime} 20.1^{\prime \prime} \mathrm{N}$ & 577 & valley & $180^{\circ}$ & \\
\hline JUN & Jungfraujoch & $7^{\circ} 59^{\prime} 07.2^{\prime \prime} \mathrm{E}$ & $46^{\circ} 32^{\prime} 50.8^{\prime \prime} \mathrm{N}$ & 3580 & mtohill & $40^{\circ}$ & \\
\hline KLO & Zürich / Kloten & $8^{\circ} 32^{\prime} 09.9^{\prime \prime} \mathrm{E}$ & $47^{\circ} 28^{\prime} 46.6^{\prime \prime} \mathrm{N}$ & 426 & flat & $135^{\circ}$ & \\
\hline KOP & Koppigen & $7^{\circ} 36^{\prime} 19.7^{\prime \prime} \mathrm{E}$ & $47^{\circ} 07^{\prime} 07.9^{\prime \prime} \mathrm{N}$ & 484 & flat & $135^{\circ}$ & \\
\hline LAE & Lägern & $8^{\circ} 23^{\prime} 50.0^{\prime \prime} \mathrm{E}$ & $47^{\circ} 28^{\prime} 55.0^{\prime \prime} \mathrm{N}$ & 845 & mtohill & $270^{\circ}$ & \\
\hline LAG & Langnau i.E. & $7^{\circ} 48^{\prime} 23.2^{\prime \prime} \mathrm{E}$ & $46^{\circ} 56^{\prime} 22.7^{\prime \prime} \mathrm{N}$ & 745 & slope & $110^{\circ}$ & $20^{\circ}$ \\
\hline LCK & Lucketen & $7^{\circ} 31^{\prime} 43.2^{\prime \prime} \mathrm{E}$ & $46^{\circ} 58^{\prime} 40.1^{\prime \prime} \mathrm{N}$ & 941 & slope & & $330^{\circ}$ \\
\hline LEI & Leibstadt & $8^{\circ} 11^{\prime} 17.7^{\prime \prime} \mathrm{E}$ & $47^{\circ} 35^{\prime} 50.2^{\prime \prime} \mathrm{N}$ & 341 & flat & $250^{\circ}$ & \\
\hline LUG & Lugano & $8^{\circ} 57^{\prime} 37.1^{\prime \prime} \mathrm{E}$ & $46^{\circ} 00^{\prime} 15.2^{\prime \prime} \mathrm{N}$ & 273 & flat & $30^{\circ}$ & \\
\hline LUZ & Luzern & $8^{\circ} 18^{\prime} 03.5^{\prime \prime} \mathrm{E}$ & $47^{\circ} 02^{\prime} 11.2^{\prime \prime} \mathrm{N}$ & 454 & flat & $180^{\circ}$ & \\
\hline MAG & Magadino / Cadenazzo & $8^{\circ} 56^{\prime} 01.2^{\prime \prime} \mathrm{E}$ & $46^{\circ} 09^{\prime} 36.1^{\prime \prime} \mathrm{N}$ & 203 & flat & $80^{\circ}$ & \\
\hline MAH & Mathod & $6^{\circ} 34^{\prime} 04.7^{\prime \prime} \mathrm{E}$ & $46^{\circ} 44^{\prime} 13.1^{\prime \prime} \mathrm{N}$ & 437 & flat & $225^{\circ}$ & \\
\hline MAS & Marsens & $7^{\circ} 04^{\prime} 10.8^{\prime \prime} \mathrm{E}$ & $46^{\circ} 39^{\prime} 23.3^{\prime \prime} \mathrm{N}$ & 714 & flat & $200^{\circ}$ & $290^{\circ}$ \\
\hline MER & Meiringen & $8^{\circ} 10^{\prime} 09.3^{\prime \prime} \mathrm{E}$ & $46^{\circ} 43^{\prime} 56.0^{\prime \prime} \mathrm{N}$ & 588 & flat & $135^{\circ}$ & \\
\hline MLS & Le Moléson & $7^{\circ} 01^{\prime} 04.1^{\prime \prime} \mathrm{E}$ & $46^{\circ} 32^{\prime} 46.2^{\prime \prime} \mathrm{N}$ & 1974 & mtohill & $0^{\circ}$ & \\
\hline MOA & Mosen & $8^{\circ} 13^{\prime} 58.1^{\prime \prime} \mathrm{E}$ & $47^{\circ} 14^{\prime} 37.8^{\prime \prime} \mathrm{N}$ & 453 & flat & $135^{\circ}$ & \\
\hline MOE & Möhlin & $7^{\circ} 52^{\prime} 40.5^{\prime \prime} \mathrm{E}$ & $47^{\circ} 34^{\prime} 19.9^{\prime \prime} \mathrm{N}$ & 344 & flat & $110^{\circ}$ & \\
\hline MRP & Monte Rosa-Plattje & $7^{\circ} 48^{\prime} 52.5^{\prime \prime} \mathrm{E}$ & $45^{\circ} 57^{\prime} 23.9^{\prime \prime} \mathrm{N}$ & 2885 & slope & & $135^{\circ}$ \\
\hline MSK & Mühleberg / Stockeren & $8^{\circ} 38^{\prime} 57.1^{\prime \prime} \mathrm{E}$ & $47^{\circ} 28^{\prime} 01.9^{\prime \prime} \mathrm{N}$ & 592 & mtohill & $270^{\circ}$ & \\
\hline MTR & Matro & $8^{\circ} 55^{\prime} 29.1^{\prime \prime} \mathrm{E}$ & $46^{\circ} 24^{\prime} 36.4^{\prime \prime} \mathrm{N}$ & 2171 & mtohill & $310^{\circ}$ & \\
\hline MUB & Mühleberg & $7^{\circ} 16^{\prime} 41.3^{\prime \prime} \mathrm{E}$ & $46^{\circ} 58^{\prime} 23.7^{\prime \prime} \mathrm{N}$ & 479 & valley & $90^{\circ}$ & $180^{\circ}$ \\
\hline MVE & Montana & $7^{\circ} 27^{\prime} 38.8^{\prime \prime} \mathrm{E}$ & $46^{\circ} 17^{\prime} 55.5^{\prime \prime} \mathrm{N}$ & 1427 & slope & & $80^{\circ}$ \\
\hline NAP & Napf & $7^{\circ} 56^{\prime} 24.2^{\prime \prime} \mathrm{E}$ & $47^{\circ} 00^{\prime} 16.8^{\prime \prime} \mathrm{N}$ & 1403 & mtohill & $270^{\circ}$ & \\
\hline NAS & Naluns / Schlivera & $10^{\circ} 15^{\prime} 41.0^{\prime \prime} \mathrm{E}$ & $46^{\circ} 49^{\prime} 01.8^{\prime \prime} \mathrm{N}$ & 2400 & mtohill & $315^{\circ}$ & \\
\hline NEE & Neerach & $8^{\circ} 38^{\prime} 57.1^{\prime \prime} \mathrm{E}$ & $47^{\circ} 28^{\prime} 01.9^{\prime \prime} \mathrm{N}$ & 592 & flat & $200^{\circ}$ & $290^{\circ}$ \\
\hline NEU & Neuchâtel & $6^{\circ} 57^{\prime} 14.8^{\prime \prime} \mathrm{E}$ & $47^{\circ} 00^{\prime} 01.6^{\prime \prime} \mathrm{N}$ & 485 & mtohill & $45^{\circ}$ & \\
\hline ORO & Oron & $6^{\circ} 51^{\prime} 29.6^{\prime \prime} \mathrm{E}$ & $46^{\circ} 34^{\prime} 20.1^{\prime \prime} \mathrm{N}$ & 827 & flat & $225^{\circ}$ & \\
\hline OTL & Locarno / Monti & $8^{\circ} 47^{\prime} 14.7^{\prime \prime} \mathrm{E}$ & $46^{\circ} 10^{\prime} 21.2^{\prime \prime} \mathrm{N}$ & 366 & slope & & $320^{\circ}$ \\
\hline PAY & Payerne & $6^{\circ} 56^{\prime} 32.7^{\prime \prime} \mathrm{E}$ & $46^{\circ} 48^{\prime} 41.7^{\prime \prime} \mathrm{N}$ & 490 & slope & $220^{\circ}$ & $310^{\circ}$ \\
\hline PIL & Pilatus & $8^{\circ} 15^{\prime} 08.4^{\prime \prime} \mathrm{E}$ & $46^{\circ} 58^{\prime} 43.9^{\prime \prime} \mathrm{N}$ & 2106 & mtohill & $225^{\circ}$ & \\
\hline $\mathrm{PIO}$ & Piotta & $8^{\circ} 41^{\prime} 17.3^{\prime \prime} \mathrm{E}$ & $46^{\circ} 30^{\prime} 53.2^{\prime \prime} \mathrm{N}$ & 990 & valley & $270^{\circ}$ & $0^{\circ}$ \\
\hline PLF & Plaffeien & $7^{\circ} 15^{\prime} 57.6^{\prime \prime} \mathrm{E}$ & $46^{\circ} 44^{\prime} 51.6^{\prime \prime} \mathrm{N}$ & 1042 & mtohill & $225^{\circ}$ & \\
\hline PMA & Piz Martegnas & $9^{\circ} 31^{\prime} 46.4^{\prime \prime} \mathrm{E}$ & $46^{\circ} 34^{\prime} 37.9^{\prime \prime} \mathrm{N}$ & 2670 & mtohill & $225^{\circ}$ & \\
\hline PRE & St-Prex & $6^{\circ} 26^{\prime} 34.8^{\prime \prime} \mathrm{E}$ & $46^{\circ} 29^{\prime} 01.2^{\prime \prime} \mathrm{N}$ & 425 & mtohill & $180^{\circ}$ & \\
\hline PSI & PSI Würenlingen & $7^{\circ} 16^{\prime} 58.7^{\prime \prime} \mathrm{E}$ & $46^{\circ} 57^{\prime} 07.6^{\prime \prime} \mathrm{N}$ & 775 & valley & $225^{\circ}$ & $135^{\circ}$ \\
\hline PUY & Pully & $6^{\circ} 40^{\prime} 02.7^{\prime \prime} \mathrm{E}$ & $46^{\circ} 30^{\prime} 44.3^{\prime \prime} \mathrm{N}$ & 455 & slope & $130^{\circ}$ & $40^{\circ}$ \\
\hline QUI & Quinten & $9^{\circ} 12^{\prime} 57.8^{\prime \prime} \mathrm{E}$ & $47^{\circ} 07^{\prime} 43.5^{\prime \prime} \mathrm{N}$ & 419 & flat & $260^{\circ}$ & $350^{\circ}$ \\
\hline RAG & Bad Ragaz & $9^{\circ} 30^{\prime} 09.3^{\prime \prime} \mathrm{E}$ & $47^{\circ} 00^{\prime} 59.8^{\prime \prime} \mathrm{N}$ & 496 & valley & $150^{\circ}$ & \\
\hline
\end{tabular}


Table S3. (continued)

\begin{tabular}{|c|c|c|c|c|c|c|c|}
\hline Code & Location & Longitude & Latitude & Elevation (m) & Classification & Valley Dir. & Slope Angle \\
\hline ROB & Poschiavo / Robbia & $10^{\circ} 03^{\prime} 40.2^{\prime \prime} \mathrm{E}$ & $46^{\circ} 20^{\prime} 47.9^{\prime \prime} \mathrm{N}$ & 1078 & slope & $200^{\circ}$ & \\
\hline RUE & Rünenberg & $7^{\circ} 52^{\prime} 45.6^{\prime \prime} \mathrm{E}$ & $47^{\circ} 26^{\prime} 04.4^{\prime \prime} \mathrm{N}$ & 611 & mtohill & $270^{\circ}$ & \\
\hline SAE & Säntis & $9^{\circ} 20^{\prime} 37.1^{\prime \prime} \mathrm{E}$ & $47^{\circ} 14^{\prime} 58.0^{\prime \prime} \mathrm{N}$ & 2502 & mtohill & $330^{\circ}$ & \\
\hline SAG & Sattel-Aegeri & $8^{\circ} 38^{\prime} 13.1^{\prime \prime} \mathrm{E}$ & $47^{\circ} 04^{\prime} 51.8^{\prime \prime} \mathrm{N}$ & 790 & valley & $45^{\circ}$ & \\
\hline SBO & Stabio & $8^{\circ} 55^{\prime} 56.5^{\prime \prime} \mathrm{E}$ & $45^{\circ} 50^{\prime} 36.2^{\prime \prime} \mathrm{N}$ & 353 & valley & $250^{\circ}$ & \\
\hline SCM & Schmerikon & $8^{\circ} 56^{\prime} 24.9^{\prime \prime} \mathrm{E}$ & $47^{\circ} 13^{\prime} 28.6^{\prime \prime} \mathrm{N}$ & 408 & flat & $90^{\circ}$ & $0^{\circ}$ \\
\hline SCU & Scuol & $10^{\circ} 16^{\prime} 59.6^{\prime \prime} \mathrm{E}$ & $46^{\circ} 47^{\prime} 35.8^{\prime \prime} \mathrm{N}$ & 1303 & slope & $330^{\circ}$ & $240^{\circ}$ \\
\hline SHA & Schaffhausen & $8^{\circ} 37^{\prime} 12.3^{\prime \prime} \mathrm{E}$ & $47^{\circ} 41^{\prime} 23.2^{\prime \prime} \mathrm{N}$ & 438 & valley & $170^{\circ}$ & $280^{\circ}$ \\
\hline SIA & Segl-Maria & $9^{\circ} 45^{\prime} 44.3^{\prime \prime} \mathrm{E}$ & $46^{\circ} 25^{\prime} 56.4^{\prime \prime} \mathrm{N}$ & 1804 & flat & $240^{\circ}$ & \\
\hline SMM & Sta. Maria, Val Müstair & $10^{\circ} 25^{\prime} 34.7^{\prime \prime} \mathrm{E}$ & $46^{\circ} 36^{\prime} 08.1^{\prime \prime} \mathrm{N}$ & 1383 & slope & $225^{\circ}$ & $135^{\circ}$ \\
\hline SPF & Schüpfheim & $8^{\circ} 00^{\prime} 44.8^{\prime \prime} \mathrm{E}$ & $46^{\circ} 56^{\prime} 49.3^{\prime \prime} \mathrm{N}$ & 742 & slope & $200^{\circ}$ & $110^{\circ}$ \\
\hline SRS & Schiers & $9^{\circ} 40^{\prime} 05.0^{\prime \prime} \mathrm{E}$ & $46^{\circ} 58^{\prime} 31.8^{\prime \prime} \mathrm{N}$ & 626 & valley & $110^{\circ}$ & $20^{\circ}$ \\
\hline STC & St. Chrischona & $8^{\circ} 28^{\prime} 44.1^{\prime \prime} \mathrm{E}$ & $47^{\circ} 29^{\prime} 59.5^{\prime \prime} \mathrm{N}$ & 410 & slope & $270^{\circ}$ & $180^{\circ}$ \\
\hline STG & St. Gallen & $9^{\circ} 23^{\prime} 54.5^{\prime \prime} \mathrm{E}$ & $47^{\circ} 25^{\prime} 31.6^{\prime \prime} \mathrm{N}$ & 775 & slope & & $135^{\circ}$ \\
\hline STK & Steckborn & $8^{\circ} 58^{\prime} 53.2^{\prime \prime} \mathrm{E}$ & $47^{\circ} 40^{\prime} 07.1^{\prime \prime} \mathrm{N}$ & 398 & flat & $45^{\circ}$ & $135^{\circ}$ \\
\hline TAE & Aadorf / Tänikon & $8^{\circ} 54^{\prime} 17.6^{\prime \prime} \mathrm{E}$ & $47^{\circ} 28^{\prime} 47.5^{\prime \prime} \mathrm{N}$ & 539 & valley & $115^{\circ}$ & \\
\hline THU & Thun & $7^{\circ} 35^{\prime} 06.8^{\prime \prime} \mathrm{E}$ & $46^{\circ} 44^{\prime} 59.5^{\prime \prime} \mathrm{N}$ & 570 & flat & $150^{\circ}$ & \\
\hline TIT & Titlis & $8^{\circ} 25^{\prime} 32.9^{\prime \prime} \mathrm{E}$ & $46^{\circ} 46^{\prime} 13.8^{\prime \prime} \mathrm{N}$ & 3040 & mtohill & $270^{\circ}$ & $135^{\circ}$ \\
\hline TSG & Arosa / Tschuggen & $9^{\circ} 39^{\prime} 58.8^{\prime \prime} \mathrm{E}$ & $46^{\circ} 46^{\prime} 51.4^{\prime \prime} \mathrm{N}$ & 1840 & mtohill & $270^{\circ}$ & $0^{\circ}$ \\
\hline UEB & Uetliberg & $8^{\circ} 33^{\prime} 24.7^{\prime \prime} \mathrm{E}$ & $47^{\circ} 28^{\prime} 59.5^{\prime \prime} \mathrm{N}$ & 496 & mtohill & $0^{\circ}$ & \\
\hline WSLBAF & Beatenberg Freiland & $7^{\circ} 46^{\prime} 18.2^{\prime \prime} \mathrm{E}$ & $46^{\circ} 42^{\prime} 02.1^{\prime \prime} \mathrm{N}$ & 1560 & slope & & $0^{\circ}$ \\
\hline WSLBTF & Bettlachstock Freiland & $7^{\circ} 25^{\prime} 02.8^{\prime \prime} \mathrm{E}$ & $47^{\circ} 13^{\prime} 28.2^{\prime \prime} \mathrm{N}$ & 1100 & slope & $225^{\circ}$ & $315^{\circ}$ \\
\hline WSLCIF & Chironico Freiland & $8^{\circ} 48^{\prime} 46.9^{\prime \prime} \mathrm{E}$ & $46^{\circ} 26^{\prime} 47.0^{\prime \prime} \mathrm{N}$ & 1380 & slope & $270^{\circ}$ & $180^{\circ}$ \\
\hline WSLCLF & Celerina Freiland & $9^{\circ} 53^{\prime} 18.2^{\prime \prime} \mathrm{E}$ & $46^{\circ} 29^{\prime} 33.1^{\prime \prime} \mathrm{N}$ & 1865 & slope & $135^{\circ}$ & $225^{\circ}$ \\
\hline WSLISF & Isone Freiland & $9^{\circ} 00^{\prime} 25.1^{\prime \prime} \mathrm{E}$ & $46^{\circ} 07^{\prime} 34.4^{\prime \prime} \mathrm{N}$ & 1149 & slope & $120^{\circ}$ & $210^{\circ}$ \\
\hline WSLJUF & Jussy Freiland & $6^{\circ} 17^{\prime} 12.6^{\prime \prime} \mathrm{E}$ & $46^{\circ} 13^{\prime} 43.1^{\prime \prime} \mathrm{N}$ & 497 & slope & $225^{\circ}$ & $135^{\circ}$ \\
\hline WSLLAF & Lausanne Freiland & $6^{\circ} 38^{\prime} 56.4^{\prime \prime} \mathrm{E}$ & $46^{\circ} 33^{\prime} 33.4^{\prime \prime} \mathrm{N}$ & 790 & mtohill & $40^{\circ}$ & \\
\hline WSLNAF & Nationalpark Freiland & $10^{\circ} 13^{\prime} 47.8^{\prime \prime} \mathrm{E}$ & $46^{\circ} 39^{\prime} 44.5^{\prime \prime} \mathrm{N}$ & 1899 & slope & $110^{\circ}$ & $315^{\circ}$ \\
\hline WSLNEF & Neunkrich Freiland & $8^{\circ} 31^{\prime} 34.8^{\prime \prime} \mathrm{E}$ & $47^{\circ} 41^{\prime} 23.3^{\prime \prime} \mathrm{N}$ & 463 & mtohill & $60^{\circ}$ & \\
\hline WSLNOF & Novaggio Freiland & $8^{\circ} 50^{\prime} 04.7^{\prime \prime} \mathrm{E}$ & $46^{\circ} 01^{\prime} 28.8^{\prime \prime} \mathrm{N}$ & 1055 & slope & & $330^{\circ}$ \\
\hline WSLOTF & Othmarsingen Freiland & $8^{\circ} 13^{\prime} 23.1^{\prime \prime} \mathrm{E}$ & $47^{\circ} 24^{\prime} 00.8^{\prime \prime} \mathrm{N}$ & 461 & mtohill & & $90^{\circ}$ \\
\hline WSLSCF & Schänis Freiland & $9^{\circ} 03^{\prime} 34.4^{\prime \prime} \mathrm{E}$ & $47^{\circ} 09^{\prime} 04.4^{\prime \prime} \mathrm{N}$ & 626 & slope & & $45^{\circ}$ \\
\hline WSLVOF & Vordemwald Freiland & $7^{\circ} 54^{\prime} 39.8^{\prime \prime} \mathrm{E}$ & $47^{\circ} 16^{\prime} 08.4^{\prime \prime} \mathrm{N}$ & 480 & slope & & $250^{\circ}$ \\
\hline WSLVSF & Visp Freiland & $7^{\circ} 50^{\prime} 33.4^{\prime \prime} \mathrm{E}$ & $46^{\circ} 18^{\prime} 09.7^{\prime \prime} \mathrm{N}$ & 640 & flat & $100^{\circ}$ & \\
\hline WYN & Wynau & $7^{\circ} 47^{\prime} 14.6^{\prime \prime} \mathrm{E}$ & $47^{\circ} 15^{\prime} 18.1^{\prime \prime} \mathrm{N}$ & 422 & valley & $225^{\circ}$ & $135^{\circ}$ \\
\hline ZER & Zermatt & $7^{\circ} 45^{\prime} 11.2^{\prime \prime} \mathrm{E}$ & $46^{\circ} 01^{\prime} 44.9^{\prime \prime} \mathrm{N}$ & 1638 & slope & $225^{\circ}$ & $315^{\circ}$ \\
\hline
\end{tabular}



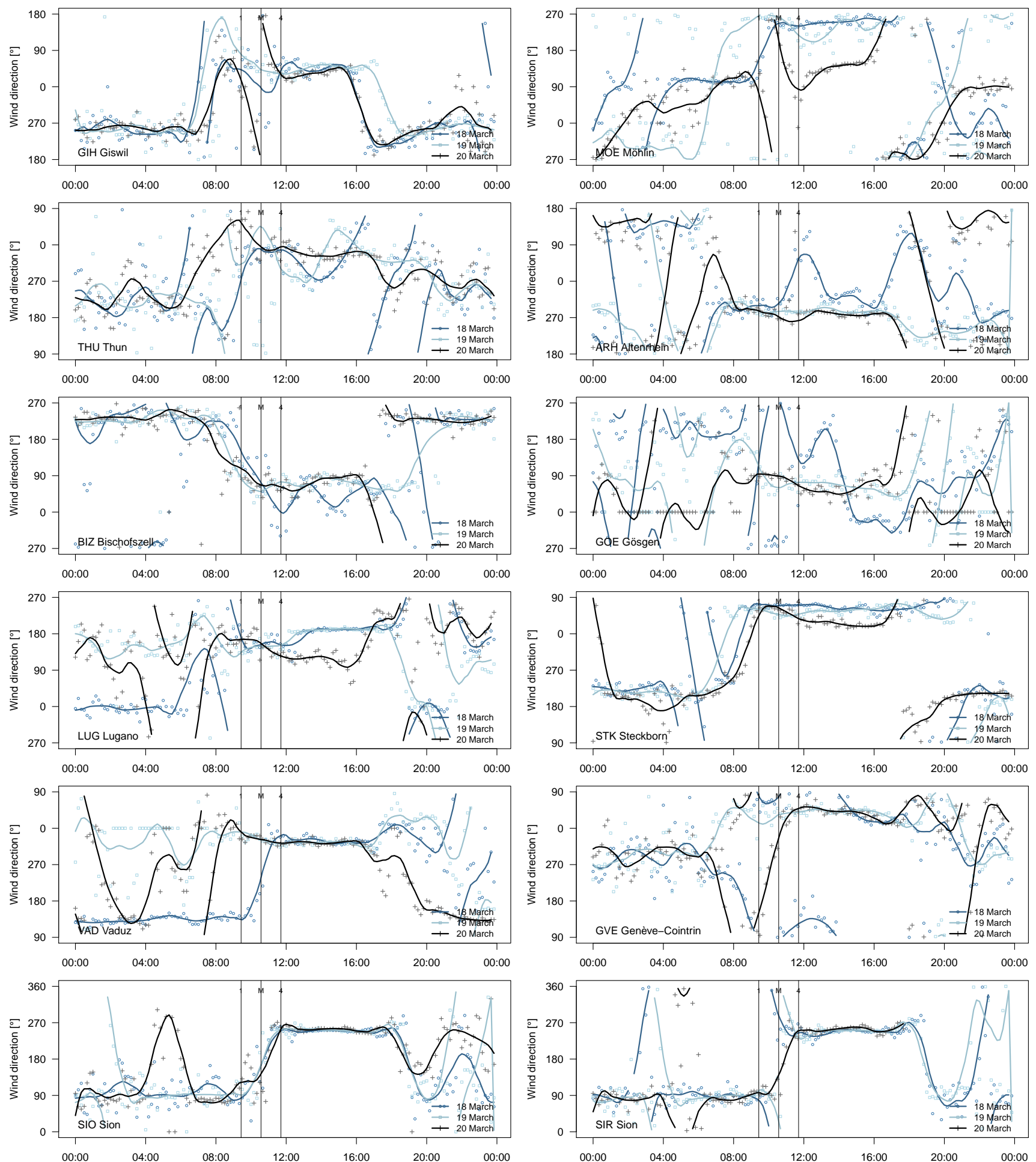

Figure S1. Wind direction evolution at MeteoSwiss sites classified as 'flat'. Black line and gray symbols: day of eclipse (20 March 2015). Light blue line and symbols (19 March 2015) and dark blue line and symbols (18 March 2015): conditions on the days before for comparison. The timing of the first (1) and last (4) contact and the eclipse maximum (M) are shown with vertical lines. Site names and codes correspond to those given in Table S3. The bold lines are local polynomial regression (loess) fits. Note that the y-axis was shifted by $0,90,180$ or $270^{\circ}$ depending on site. 

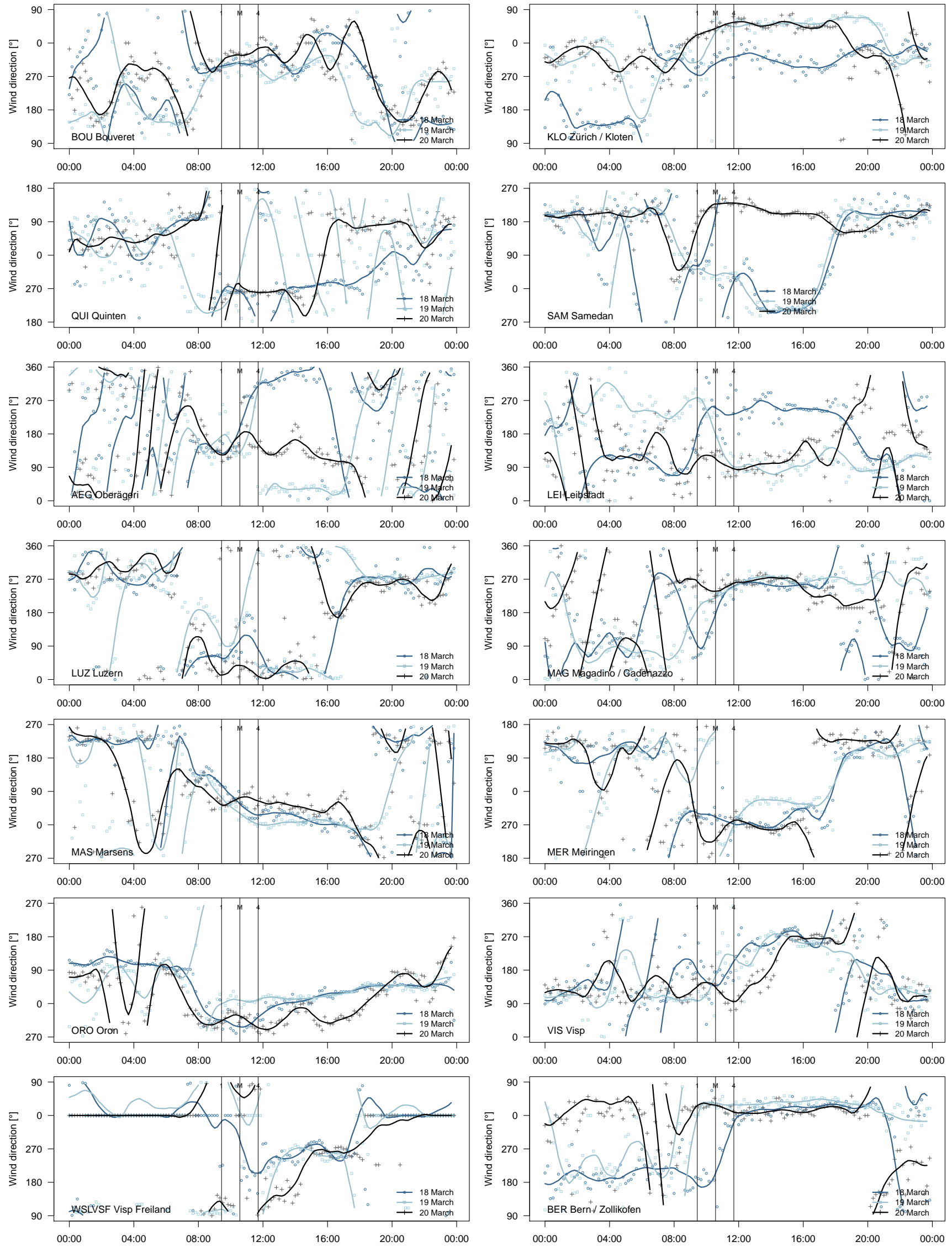

Figure S1. (continued) 

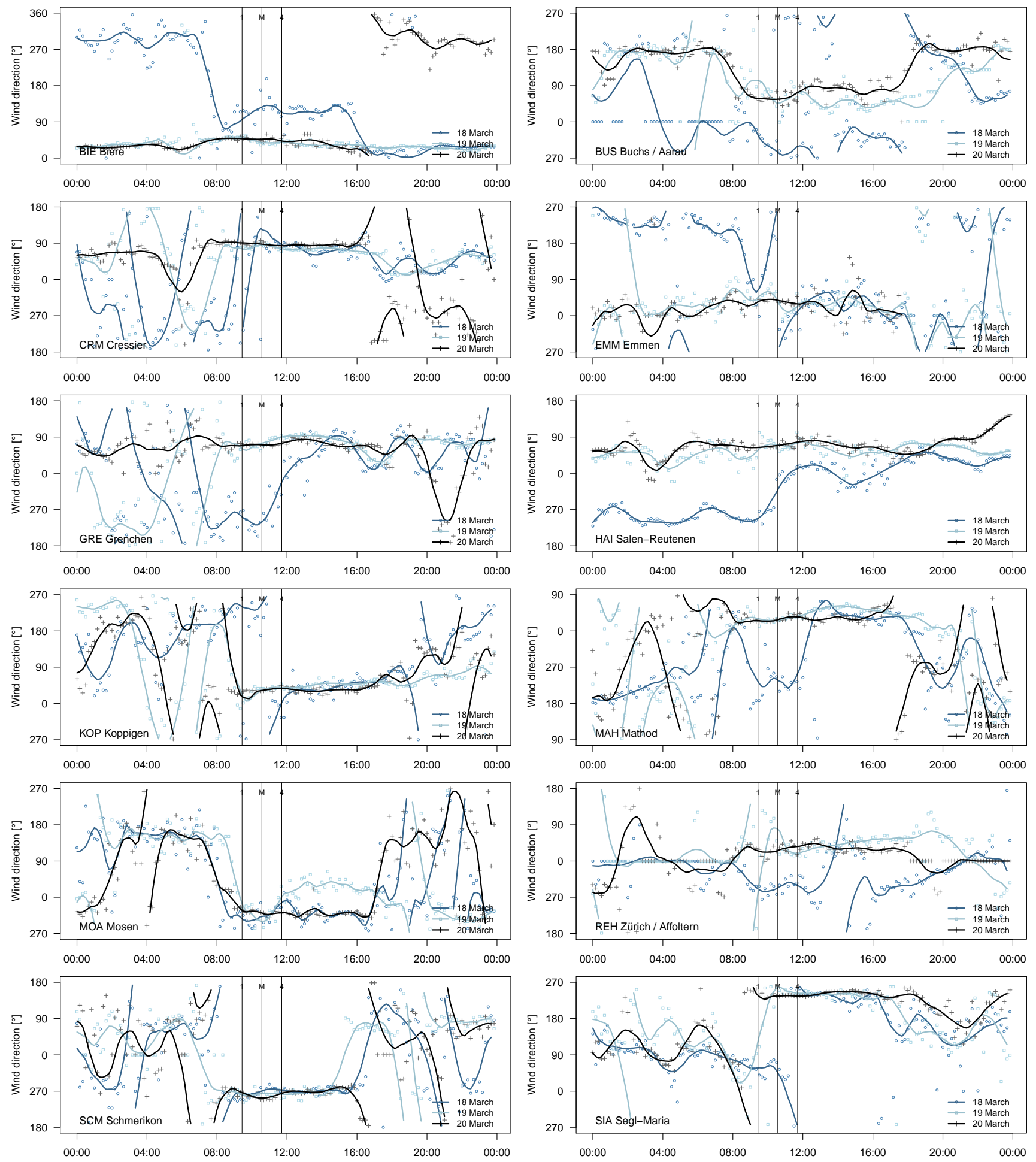

Figure S1. (continued) 

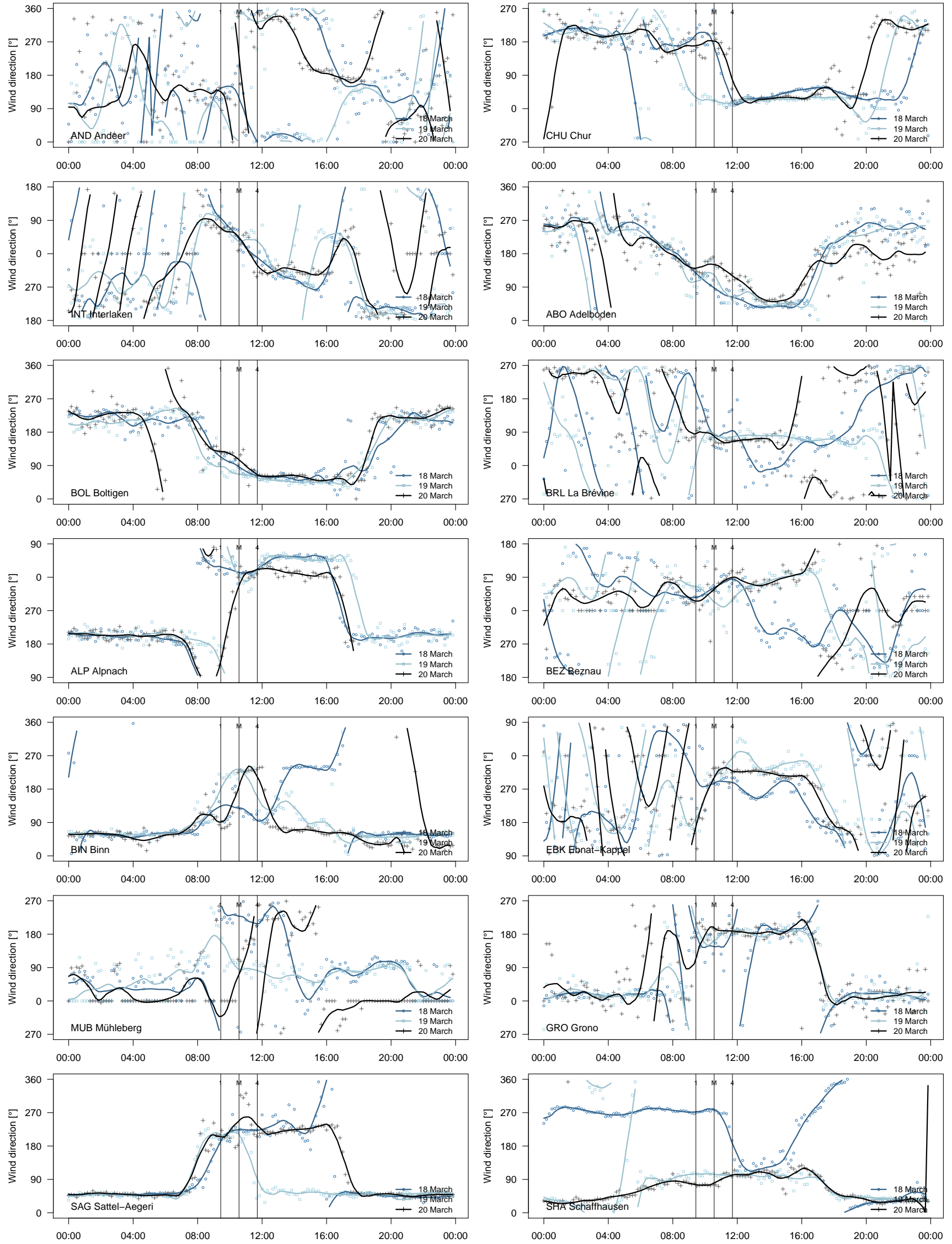

Figure S2. As Figure S1, but for sites classified as 'valley' sites in rather small valleys (otherwise a site was classified as 'flat'). 

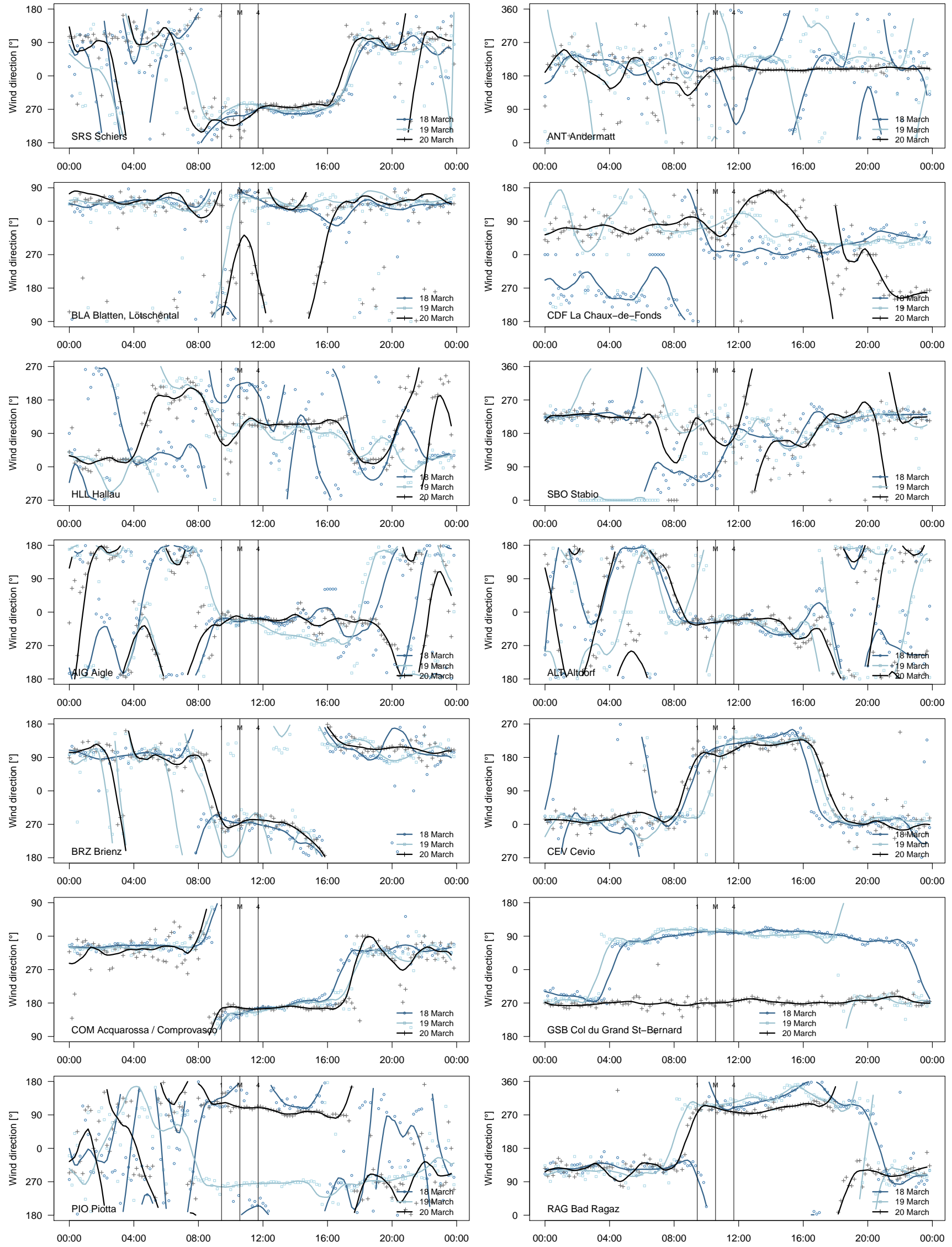

Figure S2. (continued) 

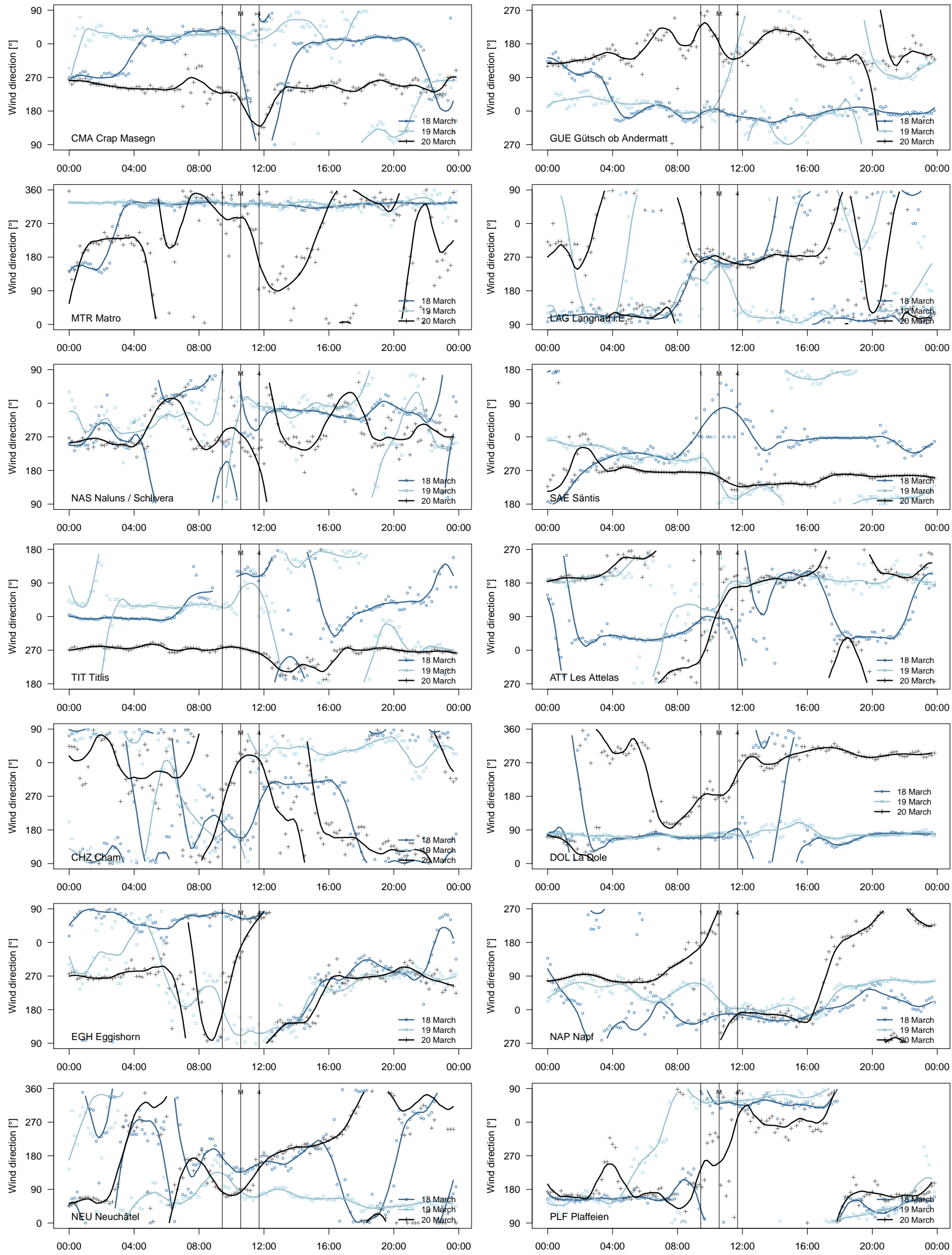

Figure S3. As Figure S1, but for sites classified as 'mountain or hilltop' sites. 

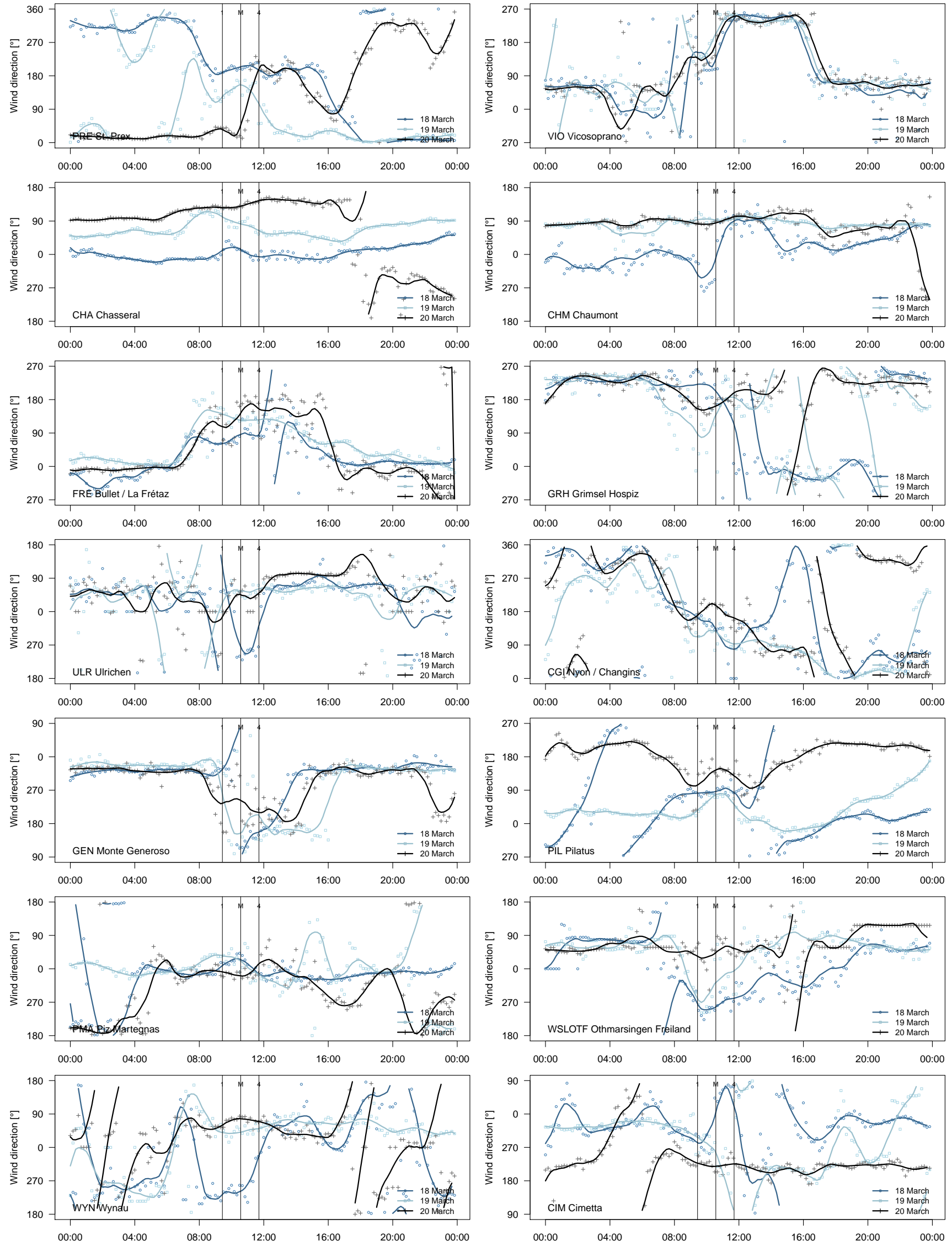

Figure S3. (continued) 

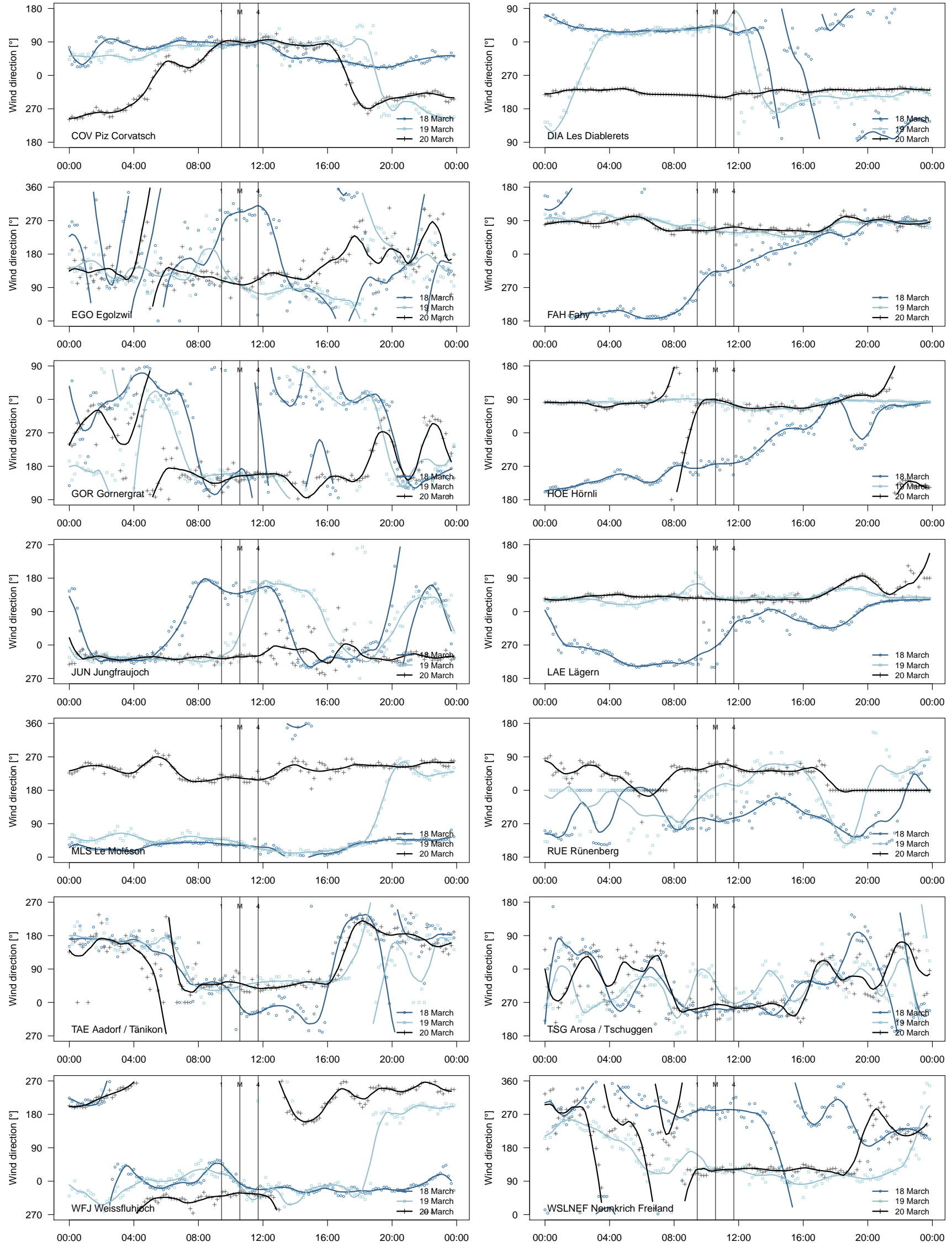

Figure S3. (continued) 

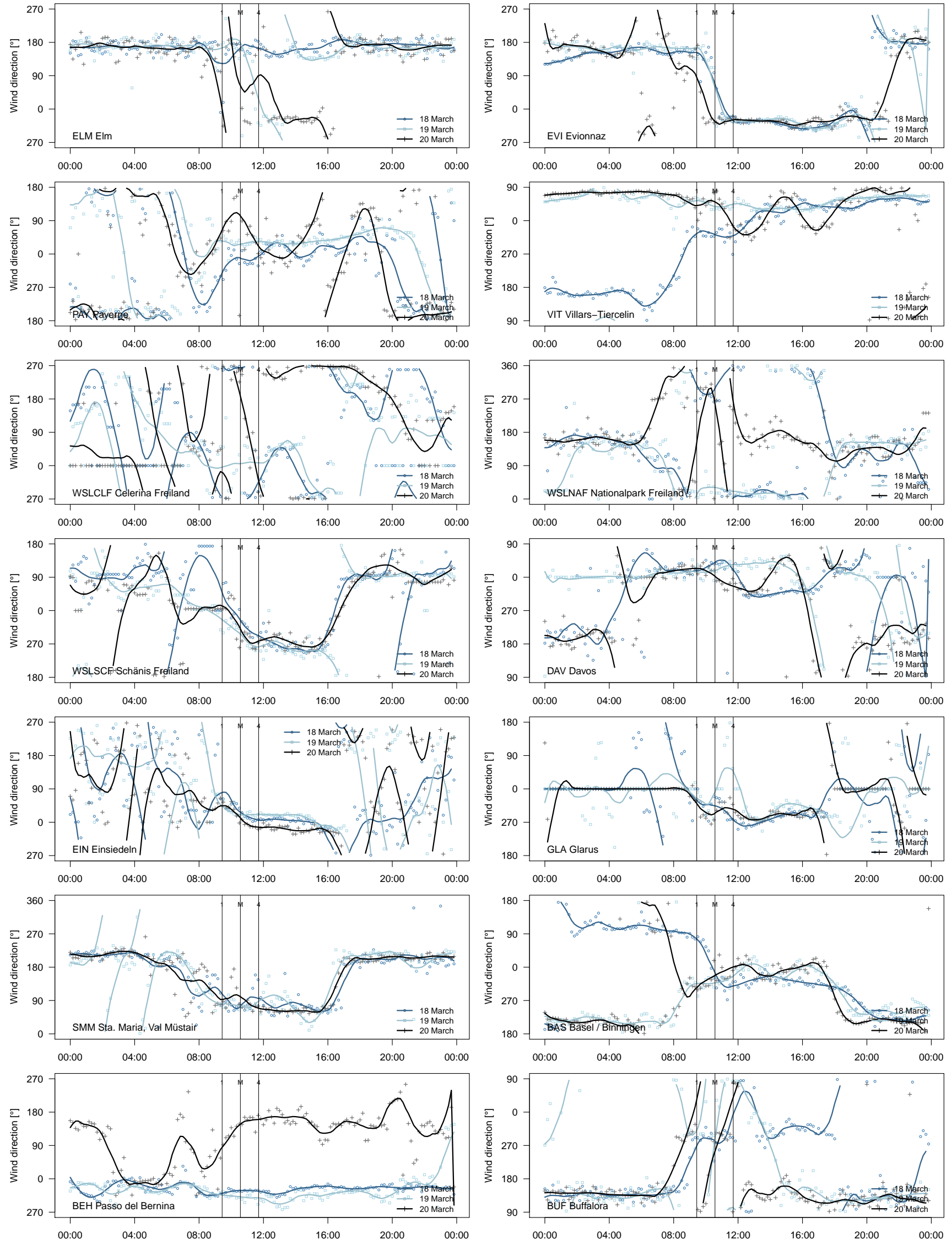

Figure S4. As Figure S1, but for sites classified as 'slope' sites. 

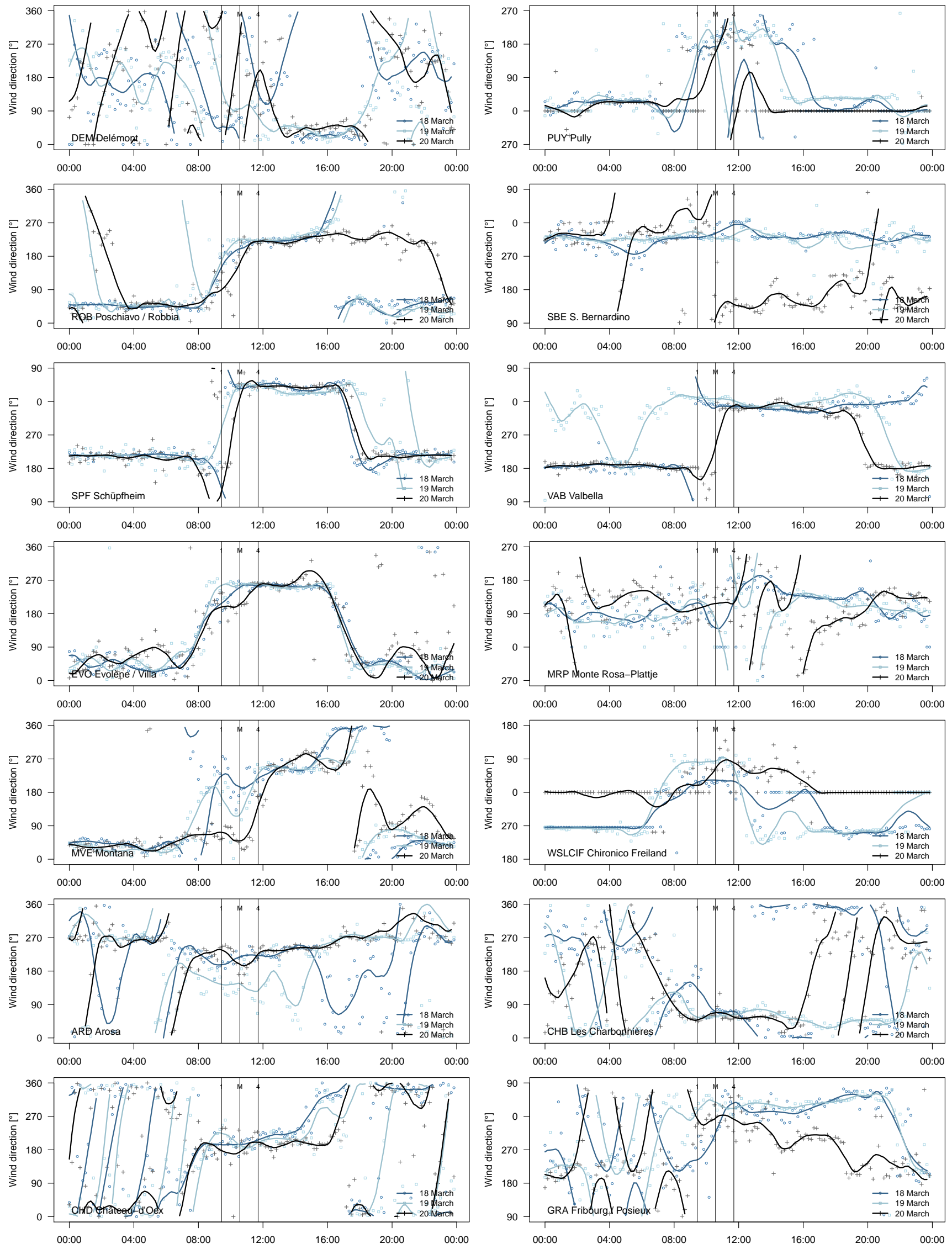

Figure S4. (continued) 

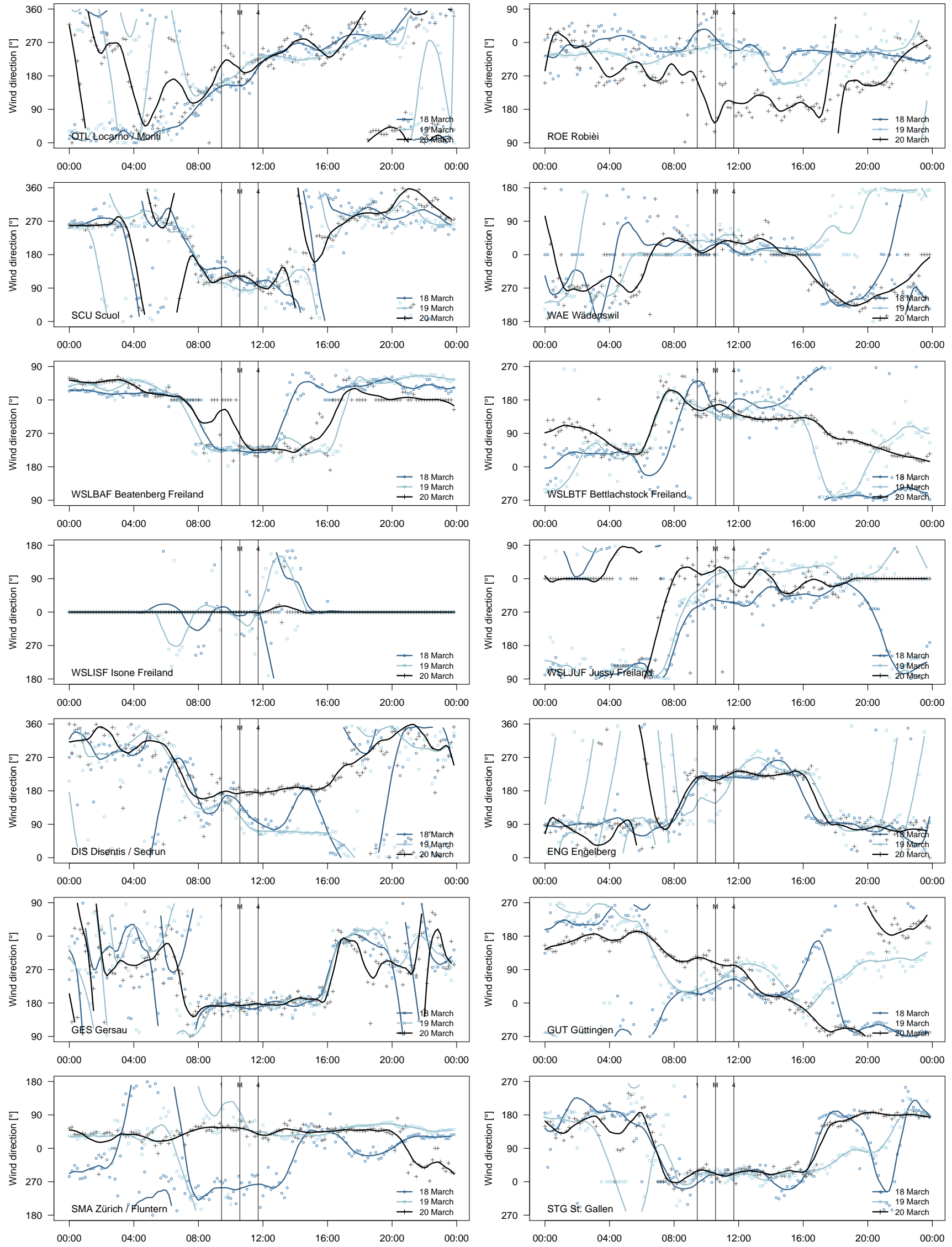

Figure S4. (continued) 

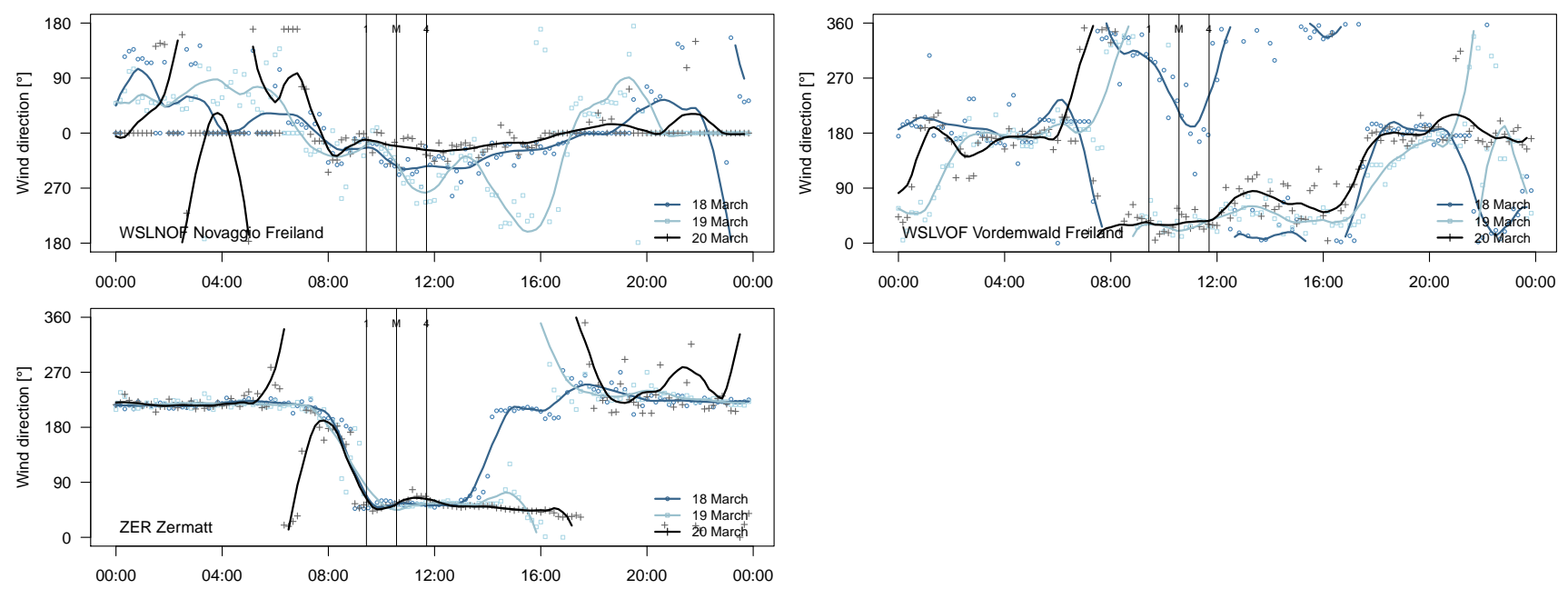

Figure S4. (continued)

\section{Remarks}

1. Some sites in Figures S1-S4 show a constant wind direction $\left(0^{\circ}, 270^{\circ}\right)$ if the wind vane was blocked during low-wind or other blocking conditions, namely at night.

2. The span for the loess fits (bold lines in Figures S1-S4) was set to 4 hours

5 3. The decision where to interrupt the loess fit lines was made automatically: if the magnitude of directional change between two data points was $200^{\circ}$ or more, then the line was interrupted. Lines were also interrupted when data were missing. 